\title{
A Generalist Predator Regulating Spread of a Wildlife Disease: Exploring Two Infection Transmission Scenarios
}

\author{
M. Sen ${ }^{1}$, M. Banerjee ${ }^{2}$, A. Morozov ${ }^{3,4}$ \\ ${ }^{1}$ Supercomputer Education and Research Centre, Indian Institute of Science, Bangalore \\ 2 Department of Mathematics and Statistics, I. I. T. Kanpur, India \\ ${ }^{3}$ Department of Mathematics, University of Leicester, UK \\ ${ }^{4}$ Shirshov Institute of Oceanology, Moscow, Russia
}

\begin{abstract}
Ecoepidemiology is a well-developed branch of theoretical ecology, which explores interplay between the trophic interactions and the disease spread. In most ecoepidemiological models, however, the authors assume the predator to be a specialist, which consumes only a single prey species. In few existing papers, in which the predator was suggested to be a generalist, the alternative food supply was always considered to be constant. This is obviously a simplification of reality, since predators can often choose between a number of different prey. Consumption of these alternative prey can dramatically change their densities and strongly influence the model predictions. In this paper, we try to bridge the gap and explore a generic ecoepidemiological system with a generalist predator, where the densities of all prey are dynamical variables. The model consists of two prey species, one of which is subject to an infectious disease, and a predator, which consumes both prey species. We investigate two main scenarios of infection transmission mode: (i) the disease transmission rate is predator independent and (ii) the transmission rate is a function of predator density. For both scenarios we fulfil an extensive bifurcation analysis. We show that including a second dynamical prey in the system can drastically change the dynamics of the single prey case. In particular, the presence of a second prey impedes disease spread by decreasing the basic reproduction number and can result in a substantial drop of the disease prevalence. We demonstrate that with efficient consumption of the second prey species by the predator, the predator-dependent disease transmission can not destabilize interactions, as in the case with a specialist predator. Interestingly, even if the population of the second prey eventually vanishes and only one prey species finally remains, the system with two prey species may exhibit different properties to those of the single prey system.
\end{abstract}

Keywords and phrases: Ecoepidemiology, generalist predator, basic reproduction number, predator-prey model, predator-dependent disease transmission, disease prevalence

Mathematics Subject Classification: 35Q53, 34B20, 35G31

\footnotetext{
${ }^{*}$ Corresponding author. E-mail: malayb@iitk.ac.in (M. Banerjee)
} 


\section{Introduction}

The spread of infectious disease within wildlife populations is often affected by trophic interactions. Modelling of trophic interactions in the presence of infection is a major subject of ecoepidemiology, which is itself a rapidly growing branch of theoretical ecology. Since the first publications in this area $[3,13,34]$, hundreds of various ecoepidemiological models have been suggested so far emphasizing various aspects of the field (for a brief review, see [35]). However, in most of the previous works, the authors assume that the predator is a specialist, i.e. it can only consume one type of resource. In the few papers, which do consider the predator to be a generalist, the alternative food source was always taken to be constant ('static') and large enough to support the predator growth rate alone $[14,15,30]$. On the other hand, it is well-known that predators are often generalists and multi-prey consumption by such predators affects the densities of all the prey species they feed on $[4,27]$. Allowing the predator to feed on multiple resources can affect the predictions obtained from simple models, which assume the predator to be a specialist, a fact which has been well recognized in theoretical ecology $[8,10,12,16,19,33]$. Surprisingly enough, aside from a few exceptions [31], most ecoepidemiological models still 'avoid' exploring these more realistic scenarios involving generalist predators with dynamical prey.

In this paper, we try to bridge this gap and explore eco-epidemiological interaction in the case where the predator is a generalist and the densities of all prey species are dynamical variables. The most important difference between ecosystems with a specialist and a generalist predator is that in the former case the extinction of one prey does not necessarily result in extinction of the predator, and this can have drastic consequences for the overall system properties such as influencing the basic reproduction number of the disease. Thus it is unclear whether or not the prior modelling results will hold in the more realistic case of a generalist predator. Obviously we cannot cover the whole problem in a single paper, so here we investigate the dynamics of a somewhat generic eco-epidemiological system in the particular case where we have two prey species, which do not compete directly with each other, and a generalist predator, and the first prey alone is subject to an infectious disease.

We explore two different scenarios of infection transmission. Firstly, we suggest that the transmission rate of disease is predator independent, as is the case in all conventional models (see [35] and the references therein). Secondly, we shall analyze a more interesting scenario, where the transmission rate is predatordependent. Often a prey population can become more vulnerable to a disease in the presence of predators, with various mechanisms causing such predator-dependent disease transmission. For instance, increased susceptibility to disease may be part of the cost of induced prey defence against predators [21,23, 29]. A well-known case study is a freshwater snail which in the presence of predators spends more time hiding inside its shell. This makes the snail more vulnerable to parasites because the organism cannot expel the blood necessary for the normal functioning of its immune system [29]. Another possible mechanism for an increase in the transmission rate is the grouping of prey in the presence of predators through the formation of fish schools, avian flocks and herds of herbivores [36], which significantly increases the number of contacts $[1,5,20]$. Note that the predator-dependent transmission scenario remains highly unexplored in the current literature. An eco-epidemiological model with predator-dependent transmission was recently considered in [25], but only for the case of a specialist predator.

For both transmission scenarios, we analyze the endemic and disease-free equilibria of the system and their stability. We explore the dependence of infection spread (in terms of the basic reproduction number) and disease prevalence on the key model parameters and compare the two transmission scenarios. Our results show that the presence of a second prey can dramatically affect the model predictions obtained from the case of a specialist predator. In particular, we show that the presence of an alterative food source decreases the disease prevalence ratio and impedes the spread of infection in the case of a predatordependent transmission rate. Inclusion of a second prey may further result in stabilization of the system, which otherwise would show high amplitude oscillations. Efficient consumption of the second prey by the predator will not allow for the Allee effect in the predator population, which was previously reported in the system with a specialist predator [25]. Surprisingly, even in the case of eventual extinction of the 
alternative prey, the systems with a specialist and a generalist predator are not equivalent in terms of their dynamical properties.

The paper is organized as follows: Section 2 introduces the model equations both for predatorindependent and the predator-dependent disease transmission; in Sections 3,4 we present the bifurcation analysis of both models, and summarize the stability properties of the system in two tables - we also present typical bifurcation diagrams for variation of key model parameters; the paper ends with a summary of the main results, general discussion and conclusions (Section 5).

\section{Model Equations}

The model describes interaction of three species- two prey species and a generalist predator- by combining a standard S-I model and a two-prey one predator model. We assume that the first prey species is subject to infection and hence we divide the whole population of this species into two classes, susceptible and infected. The predator only consumes infected members of the first prey species, thus healthy individuals of this species escape from predation. This scenario is largely supported by empirical data $[7,9,17,18]$. The second prey species is an alternative resource for the predator. The overall model is a system of four coupled ordinary differential equations which describe the dynamics of susceptible prey \#1 $(S)$, infected prey \#1 $(I)$, prey \#2 $(N)$ and the generalist predator $(P)$.

$$
\begin{aligned}
\frac{d S}{d t} & =r_{1}\left(1-\frac{S+I}{K_{1}}\right) S-\lambda(P) S I, \\
\frac{d I}{d t} & =\lambda(P) S I-d_{1} I-f(I, N) P, \\
\frac{d N}{d t} & =r_{2}\left(1-\frac{N}{K_{2}}\right) N-g(I, N) P, \\
\frac{d P}{d t} & =\theta_{1} f(I, N) P+\theta_{2} g(I, N) P-d_{2} P .
\end{aligned}
$$

The state variables and the model parameters are briefly listed in Table 1.

The growth rate of each prey population is described by a logistic function, where $K_{i}$ and $r_{i}$ are, respectively, the carrying capacity and the maximal per capita growth rate of species $i$. We assume that only healthy individuals of prey \#1 can reproduce; however, the infected subpopulation still affects the overall prey growth via competition for resources and therefore contributes to the carrying capacity of the healthy subpopulation. We consider the situation where the two prey species do not compete directly for the same resource, but can affect each other by influencing predator density (known in ecology as 'apparent competition', see [32]). When feeding on the infected prey $I$, the predator is assumed not to succumb to the infection. Consumption of $I$ and $N$ is described by the functional responses $f(I, N)$ and $g(I, N)$, respectively. There exist a large number of various shapes of the multi-prey functional response [11] and choosing a particular one can affect modelling results. In this paper, we consider the simplest Holling type-I functional response (linear response) and assume that the consumption of each prey occurs independently of the other prey species (i.e. no explicit prey selectivity):

$$
f(I, N) \equiv f(I)=a I, g(I, N) \equiv g(N)=b N .
$$

Analysis of the influence of saturation in $f(I, N)$ and $g(I, N)$ on modelling results should be done elsewhere.

The disease transmission is parameterized via the classical mass action term $\lambda S I[2,24]$; this scenario is observed in situations, where the number of contacts between individuals is not fixed, but is proportional to the population size. The coefficient of proportionality $\lambda$ is called the transmission coefficient. In earlier models, the transmission coefficient was considered to be constant [35], however, a large amount of empirical evidence indicates that disease transmission can be affected by the presence of predators (see 
relevant references in the Introduction). Thus, we shall explore here both scenarios: (i) $\lambda=$ const and (ii) $\lambda=\lambda(P)$. There may be various mechanisms to describe the nature of the relationship $\lambda=\lambda(P)$; here we assume the simplest linear form of dependence (which can be considered as the first term in the Taylor expansion about the predator-free state $P=0$ ),

$$
\lambda(P) \equiv \lambda_{0}+\alpha P
$$

where $\lambda_{0}$ is the predator density independent disease transmission rate and $\alpha$ is the predator regulated additional disease transmission rate [25]. Although we can potentially have $\alpha<0$, we shall focus on the case $\alpha>0$, i.e. assuming that the presence of the predator facilitates the spread of the infection.

\begin{tabular}{cl}
\hline Variables & Description \\
\hline & Time \\
$S$ & Density of susceptible prey \#1 \\
$I$ & Density of infected prey \#1 \\
$N$ & Density of prey \#2 \\
$P$ & Density of predator \\
\hline & Description \\
\hline$r_{1}$ & Growth rate of prey \#1 \\
$K_{1}$ & Carrying capacity of prey \#1 \\
$\lambda_{0}+\alpha P$ & Transmission coefficient of the disease spread \\
$\lambda_{0}$ & Disease transmission rate, independent of predator density \\
$d_{1}$ & Predator density mediated additional disease transmission rate \\
$r_{2}$ & Disease virulence (i.e. extra mortality caused by the disease) \\
$K_{2}$ & Functional response describing the grazing of $I$ by the predator \\
$r_{1}, \theta_{2}$ & Growth rate of prey \#2 \\
$d_{2}$ & Carrying capacity of prey \#2 \\
& Functional response describing the grazing of $N$ by the predator \\
& Conversion efficiencies for consumption of prey \#1,2 \\
& Intrinsic death rate of the predator \\
\hline
\end{tabular}

TABLE 1. Description of variables and parameters present in the basic model.

It is worth mentioning that in the absence of the second prey $(N \equiv 0)$ and for a constant transmission coefficient $\lambda$, the model behavior is well understood $[25,26]$; its properties are briefly listed in the beginning of the next section. Introducing a predator-dependent transmission $\lambda=\lambda(P)$ into the model with a single prey $(N \equiv 0)$ makes it more complex, a scenario which has been considered in [25]. The major results of this investigation are briefly listed in the beginning of section 4 .

In the forthcoming two sections we will be considering model $(2.1)-(2.4)$ in the two above mentioned cases: $\alpha=0$ (Model 1) and $\alpha>0$ (Model 2). Note that using standard approaches (e.g. [30]), one can easily prove boundedness as well as positive invariance of solutions of the general model. We do not show these proofs for the sake of brevity. 


\section{Model 1: predator-independent disease transmission scenario}

For a predator-independent transmission scenario, the model equations read

$$
\begin{aligned}
\frac{d S}{d t} & =r_{1}\left(1-\frac{S+I}{K}\right) S-\lambda_{0} S I, \\
\frac{d I}{d t} & =\lambda_{0} S I-d_{1} I-a I P, \\
\frac{d N}{d t} & =r_{2}\left(1-\frac{N}{K}\right) N-b N P, \\
\frac{d P}{d t} & =\theta_{1} a I P+\theta_{2} b N P-d_{2} P .
\end{aligned}
$$

Note that without any generality loss, we can consider that $K_{1}=K_{2}=K$ since we can always re-scale the species densities to have the same carrying capacities.

Next, to better understand the role of the second prey on dynamics of the system, we shall briefly consider the same model with $N \equiv 0$. The model has the following stationary states $[25,26]$. The trivial stationary state is $(0,0,0)$; it is always unstable. The disease-free and predator-free stationary state is given by $(K, 0,0)$; it is stable for $D>K \lambda_{0}$ and unstable otherwise. The predator-free stationary state is $\left(S_{6}, I_{6}, 0\right)$, with $S_{6}=d_{1} / \lambda, I_{6}=r\left(K \lambda_{0}-d_{1}\right) /\left(\lambda_{0}\left(K \lambda_{0}+r\right)\right)$. Finally, there is a unique interior stationary state $\left(S_{8}, I_{8}, P_{8}\right)$, where the stationary values are determined by

$$
S_{8}=\frac{r K \theta_{1} a-r \delta-\delta K \lambda_{0}}{r a \theta_{1}}, I_{8}=\frac{\delta}{\theta_{1} a}, P_{8}=\frac{r K a \theta_{1} \lambda_{0}-r \delta \lambda_{0}-\delta \lambda_{0} K-a \theta_{1} r d_{1}}{a \theta_{1} r d_{1}} .
$$

This stationary state is always stable when it exists $[25,26]$.

A typical bifurcation diagram for Model 1 in the absence of the second prey is shown in Fig.1, which represents the variation in the equilibrium species densities as functions of $\lambda_{0}$. The figure is constructed for the following parameters $r_{1}=1, K=10, d_{1}=.5, a=1, \theta_{1}=.2$. From the diagram one can see that the predator can establish in the system only for intermediate values of $\lambda_{0}$. In other words, a small transmission rate $\lambda_{0}$ is not enough to spread the infection in the prey (and, consequently, the predator cannot survive since $I=0$ ), whereas a large transmission rate will reduce the population size of the infected prey to the point where it is not large enough to support the predator's growth.

Introduction of an alternative prey $N$ can drastically change the above results.

\subsection{Equilibria}

The full Model 1 (3.1)-(3.4) with a second prey has the following equilibria. There is the trivial equilibrium point $E_{0}(0,0,0,0)$, two axial equilibria $E_{1}(K, 0,0,0), E_{2}(0,0, K, 0)$ (only one prey species can survive and there is no infection in the system) and one boundary equilibrium point given by $E_{3}(K, 0, K, 0)$ (both prey species survive, there is infection in the system), which exist for any system parameters.

The model may also possess five more boundary equilibria given by $E_{4}\left(\frac{d_{1}}{\lambda_{0}}, \frac{r_{1}\left(\lambda_{0} K-d_{1}\right)}{\lambda_{0}\left(r_{1}+\lambda_{0} K\right)}, 0,0\right)$, $E_{5}\left(0,0, \frac{r_{2}\left(\theta_{2} b K-d_{2}\right)}{\theta_{2} b^{2} K}, \frac{d_{2}}{\theta_{2} b}\right), \quad E_{6}\left(\frac{d_{1}}{\lambda_{0}}, \quad \frac{r_{1}\left(\lambda_{0} K-d_{1}\right)}{\lambda_{0}\left(r_{1}+\lambda_{0} K\right)}, K, 0\right), \quad E_{7}\left(K, 0, \frac{d_{2}}{\theta_{2} b}, \frac{r_{2}\left(\theta_{2} b K-d_{2}\right)}{\theta_{2} b^{2} K}\right)$ and $E_{8}\left(\frac{\theta_{1} a r_{1} K-d_{2} r_{1}-d_{2} \lambda_{0} K}{\theta_{1} a r_{1}}, \frac{d_{2}}{\theta_{1} a}, 0, \frac{\lambda_{0} \theta_{1} a r_{1} K-\lambda_{0} d_{2} r_{1}-d_{2} \lambda_{0}^{2} K-d_{1} \theta_{1} a r_{1}}{a^{2} \theta_{1} r_{1}}\right)$ when certain parametric restrictions are satisfied, the related results are stated in the propositions below. In each of the above equilibrium at least one component is absent.

The equilibrium states with $I_{i}>0$ are called the endemic equilibria (states). Let us define the basic reproduction number for the disease transmission as follows

$$
\mathcal{R}_{0}=\frac{\lambda_{0} K}{d_{1}} .
$$




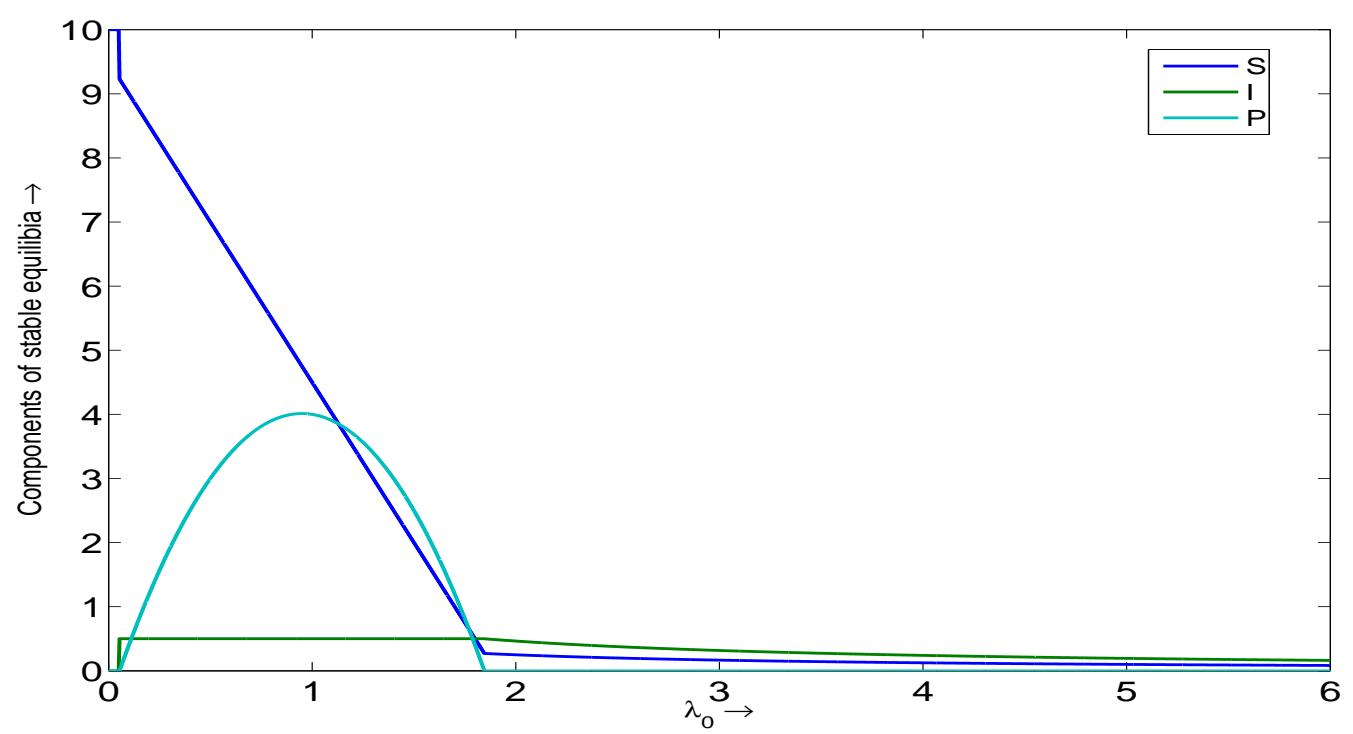

Figure 1. Equilibrium species densities in Model 1 in the absence of prey \#2 are constructed as functions of the transmission coefficient $\lambda_{0}$. The other model parameters are $r_{1}=1, K=10, d_{1}=.5, a=1, \theta_{1}=.2$.

The basic reproduction number can be interpreted in the following way [6]: $1 / d_{1}$ is the average lifespan of infected prey individuals and $\lambda_{0}$ is the rate of new infections, and hence the rate of generation of new infective individuals, when the number of infected prey individuals is assumed to be small, is given by $\frac{\lambda_{0}}{d_{1}} K$. With the help of the basic reproduction number, we can define the conditions of the existence of the system's equilibria:

Proposition 3.1. Let us define,

$$
\begin{array}{ll}
\boldsymbol{H}_{1} & : \mathcal{R}_{0}>1 . \\
\boldsymbol{H}_{2} & : \theta_{2} b K>d_{2} . \\
\boldsymbol{H}_{3} & : \theta_{1} a r_{1} K>d_{2}\left(r_{1}+\lambda_{0} K\right) . \\
\boldsymbol{H}_{\mathbf{4}} & : \theta_{1} a r_{1}\left(\lambda_{0} K-d_{1}\right)>d_{2} \lambda_{0}\left(r_{1}+\lambda_{0} K\right) .
\end{array}
$$

If $\boldsymbol{H}_{\mathbf{1}}$ is satisfied then $E_{4}$ and $E_{6}$ exist. If $\boldsymbol{H}_{\mathbf{2}}$ is satisfied then $E_{5}$ and $E_{7}$ exist. If both $\boldsymbol{H}_{\mathbf{3}}$ and $\boldsymbol{H}_{\mathbf{4}}$ hold then $E_{8}$ is feasible.

Note that the condition $\mathcal{R}_{0}>1$ is the necessary condition for the existence of the endemic stationary states $E_{4}, E_{6}$ and $E_{8}$.

Finally, the model exhibits a unique interior equilibrium point ensuring the coexistence of all four components $\left(S_{*}, I_{*}, P_{*}, N_{*}\right)$. The specific parametric restrictions required for the existence of the interior equilibrium are given in the following proposition.

Proposition 3.2. Let us define,

$$
\begin{aligned}
& \boldsymbol{H}_{\mathbf{5}}: \theta_{1} a r_{1}\left(\lambda_{0} K-d_{1}\right)+\lambda_{0}\left(r_{1}+\lambda_{0} K\right)\left(\theta_{2} b K-d_{2}\right)>0 . \\
& \boldsymbol{H}_{\mathbf{6}}: a_{2}\left(\theta_{2} b K-d_{2}\right)<\theta_{2} b^{2} K\left(\lambda_{0} K-d_{1}\right) . \\
& \boldsymbol{H}_{\mathbf{7}}: a^{2} r_{2} \theta_{1} r_{1}>b\left[\theta_{1} a r_{1}\left(\lambda_{0} K-d_{1}\right)-d_{2} \lambda_{0}\left(r_{1}+\lambda_{0} K\right)\right] .
\end{aligned}
$$

The model possesses a unique interior equilibrium point $E_{*}\left(S_{*}, I_{*}, N_{*}, P_{*}\right)$ if $\boldsymbol{H}_{\mathbf{5}}, \boldsymbol{H}_{\mathbf{6}}$ and $\boldsymbol{H}_{\mathbf{7}}$ hold. 
The components of interior equilibrium point are given by the following expressions

$$
\begin{aligned}
S_{*} & =\frac{a^{2} r_{2} \theta_{1} r_{1} K+\lambda_{0} K^{2} \theta_{2} b^{2} d_{1}-a r_{2} d_{2} r_{1}-a r_{2} d_{2} \lambda_{0} K+r_{1} \theta_{2} b a r_{2} K+r_{1} \theta_{2} b^{2} K d_{1}+\lambda_{0} K^{2} \theta_{2} b a r_{2}}{a^{2} r_{2} \theta_{1} r_{1}+r_{1} \theta_{2} b^{2} K \lambda_{0}+\lambda_{0}^{2} K^{2} \theta_{2} b^{2}}, \\
I_{*} & =\frac{r_{1}\left(\lambda_{0} K^{2} \theta_{2} b^{2}-\theta_{2} b K a r_{2}-\theta_{2} b^{2} K d_{1}+d_{2} a r_{2}\right)}{a^{2} r_{2} \theta_{1} r_{1}+r_{1} \theta_{2} b^{2} K \lambda_{0}+\lambda_{0}^{2} K^{2} \theta_{2} b^{2}}, \\
N_{*} & =\frac{K\left(a^{2} r_{2} \theta_{1} r_{1}-b \lambda_{0} a \theta_{1} r_{1} K+b \lambda_{0} d_{2} r_{1}+b \lambda_{0}^{2} d_{2} K+b d_{1} a \theta_{1} r_{1}\right)}{a^{2} r_{2} \theta_{1} r_{1}+r_{1} \theta_{2} b^{2} K \lambda_{0}+\lambda_{0}^{2} K^{2} \theta_{2} b^{2}} \\
P_{*} & =\frac{r_{2}\left(\lambda_{0} \theta_{1} a r_{1} K-\lambda_{0} d_{2} r_{1}-d_{2} \lambda_{0}^{2} K+\lambda_{0} r_{1} \theta_{2} b K+\lambda_{0}^{2} K^{2} \theta_{2} b-d_{1} \theta_{1} a r_{1}\right)}{a^{2} r_{2} \theta_{1} r_{1}+r_{1} \theta_{2} b^{2} K \lambda_{0}+\lambda_{0}^{2} K^{2} \theta_{2} b^{2}} .
\end{aligned}
$$

Note that the condition $\mathcal{R}_{0}>1$ is necessary (but not sufficient) for $\boldsymbol{H}_{\mathbf{6}}$ to hold. We can also introduce a modified basic reproduction number $\mathcal{R}_{1}$ the disease spread in the presence of the predator. To do this, we consider the per capita growth rate of $I$ when the predator density is supported by prey \#2 and is given approximately by $P_{7}=\frac{r_{2}\left(\theta_{2} b K-d_{2}\right)}{\theta_{2} b^{2} K}$ (see equilibrium $E_{7}$ ). The value of $\mathcal{R}_{1}$ is then defined as

$$
\mathcal{R}_{1}=\frac{\lambda_{0} K}{d_{1}+a P_{7}}=\frac{\lambda_{0} K}{d_{1}+\frac{r_{2}\left(\theta_{2} b K-d_{2}\right)}{\theta_{2} b^{2} K}} .
$$

Thus, a necessary condition for the existence of the endemic equilibrium $E_{*}$ is given by $\mathcal{R}_{1}>1$, which coincides with condition $\boldsymbol{H}_{6}$. Note that the condition $\mathcal{R}_{1}>1$ is more restrictive than $\mathcal{R}_{0}>1$.

The main results on system's equilibria in Model 1 are summarized in Table 2.

\subsection{Stability of equilibria}

Stability of the stationary state is determined by the eigenvalues of the linearized system. The Jacobian matrix for Model 1 evaluated at an arbitrary point is given by

$$
J=\left[\begin{array}{cccc}
r_{1}\left(1-\frac{2 S+I}{K}\right)-\lambda_{0} I & -r_{1} \frac{S}{K}-\lambda_{0} S & 0 & 0 \\
\lambda_{0} I & \lambda_{0} S-d_{1}-a P & 0 & -a I \\
0 & 0 & r_{2}\left(1-\frac{2 N}{K}\right)-b P & -b N \\
0 & \theta_{1} a P & \theta_{2} b P & \theta_{1} a I+\theta_{2} b N-d_{2}
\end{array}\right]
$$

$E_{0}(0,0,0,0)$ is trivial equilibrium point and evaluating the Jacobian matrix at $E_{0}$ we get the eigenvalues $r_{1}>0,-d_{1}<0, r_{2}>0$ and $-d_{2}<0$. Hence $E_{0}$ is a saddle-point having a two-dimensional stable manifold and a two-dimensional unstable manifold.

$E_{1}(K, 0,0,0)$ and $E_{2}(0,0, K, 0)$ are two axial equilibrium points. Evaluating the Jacobian matrix at $E_{1}$ we find the eigenvalues of the Jacobian matrix as $-r_{1}<0, \lambda_{0} K-d_{1}, r_{2}>0$ and $-d_{2}<0$. Hence $E_{1}$ is a saddle point and the dimension of the unstable manifold is 2 if $\mathcal{R}_{0}>1$ and is 1 otherwise.

The eigenvalues of $J$ at $E_{2}$ are $r_{1}>0,-d_{1}<0,-r_{2}<0$ and $\theta_{2} b K-d_{2}$. Clearly $E_{2}$ is a saddle point having a two-dimensional unstable manifold if $\theta_{2} b K>d_{2}$.

Evaluating the Jacobian matrix at the first boundary equilibrium point $E_{3}(K, 0, K, 0)$ we find its eigenvalues $-r_{1}<0, \lambda_{0} K-d_{1},-r_{2}<0$ and $\theta_{2} b K-d_{2}$. So $E_{3}$ is locally asymptotically stable if $\mathcal{R}_{0}<1$ and $\theta_{2} b K<d_{2}$. Combining these conditions we get $K<\min \left\{\frac{d_{1}}{\lambda_{0}}, \frac{d_{2}}{\theta_{2} b}\right\}$. $E_{3}$ is a saddle point having a two-dimensional unstable manifold if $\mathcal{R}_{0}>1$ and $\theta_{2} b K>d_{2}$, that is if $K>\max \left\{\frac{d_{1}}{\lambda_{0}}, \frac{d_{2}}{\theta_{2} b}\right\}$.

For $E_{4}$, the Jacobian matrix is

$$
J_{4}=\left[\begin{array}{cccc}
-\frac{r_{1} d_{1}}{\lambda_{0} K} & -\frac{r_{1} d_{1}}{\lambda_{0} K}-d_{1} & 0 & 0 \\
\frac{r_{1}\left(\lambda_{0} K-d_{1}\right)}{r_{1}+\lambda_{0} K} & 0 & 0 & -\frac{a r_{1}\left(\lambda_{0} K-d_{1}\right)}{\lambda_{0}\left(r_{1}+\lambda_{0} K\right)} \\
0 & 0 & r_{2} & 0 \\
0 & 0 & 0 & \frac{\theta_{1} a r_{1} \lambda_{0} K-\lambda_{0} d_{2} r_{1}-d_{2} \lambda_{0}^{2} K-d_{1} \theta_{1} r_{1} K}{\lambda_{0}\left(r_{1}+\lambda_{0} K\right)}
\end{array}\right]
$$


$E_{4}$ is a saddle point with a two-dimensional unstable manifold if $\theta_{1} a r_{1}\left(\lambda_{0} K-d_{1}\right)>d_{2} \lambda_{0}\left(r_{1}+\lambda_{0} K\right)$. Otherwise it will have a one-dimensional unstable manifold and a three-dimensional stable manifold.

For $E_{5}$

$$
J_{5}=\left[\begin{array}{cccc}
r_{1} & 0 & 0 & 0 \\
0 & -\frac{b^{2} \theta_{2} K d_{1}+a r_{2}\left(\theta_{2} b K-d_{2}\right)}{\left(b^{2} \theta_{2} K\right)} & 0 & 0 \\
0 & 0 & -\frac{r_{2} d_{2}}{K \theta_{2} b} & -\frac{d_{2}}{\theta_{2}} \\
0 & \frac{\theta_{1} a r_{2}\left(\theta_{2} b K-d_{2}\right)}{b^{2} \theta_{2} K} & \frac{r_{2}\left(\theta_{2} b K-d_{2}\right)}{b K} & 0
\end{array}\right]
$$

so $E_{5}$ is a saddle point with a one-dimensional unstable manifold.

For $E_{6}$

$$
J_{6}=\left[\begin{array}{cccc}
-\frac{r_{1} d_{1}}{\lambda_{0} K} & -\frac{r_{1} d_{1}}{\lambda_{0} K}-d_{1} & 0 & 0 \\
\frac{r_{1}\left(\lambda_{0} K-d_{1}\right)}{r_{1}+\lambda_{0} K} & 0 & 0 & -\frac{a r_{1}\left(\lambda_{0} K-d_{1}\right)}{\lambda_{0}\left(r_{1}+\lambda_{0} K\right)} \\
0 & 0 & -r_{2} & -b K \\
0 & 0 & 0 & \frac{\theta_{1} a r_{1}\left(\lambda_{0} K-d_{1}\right)+\lambda_{0}\left(r_{1}+\lambda_{0} K\right)\left(\theta_{2} b K-d_{2}\right)}{\lambda_{0}\left(r_{1}+\lambda_{0} K\right)}
\end{array}\right]
$$

$E_{6}$ is a stable point if $\theta_{1} a r_{1}\left(\lambda_{0} K-d_{1}\right)+\lambda_{0}\left(r_{1}+\lambda_{0} K\right)\left(\theta_{2} b K-d_{2}\right)<0$ otherwise it is a saddle point with a one-dimensional unstable manifold.

For $E_{7}$

$$
J_{7}=\left[\begin{array}{cccc}
-r_{1} & -r_{1}-\lambda_{0} K & 0 & 0 \\
0 & \frac{b^{2} \theta_{2} K\left(\lambda_{0} K-d_{1}\right)-a r_{2}\left(\theta_{2} b K-d_{2}\right)}{\left(b^{2} \theta_{2} K\right)} & 0 & 0 \\
0 & 0 & -\frac{r_{2} d_{2}}{K \theta_{2} b} & -\frac{d_{2}}{\theta_{2}} \\
0 & \frac{\theta_{1} a r_{2}\left(\theta_{2} b K-d_{2}\right)}{b^{2} \theta_{2} K} & \frac{r_{2}\left(\theta_{2} b K-d_{2}\right)}{b K} & 0
\end{array}\right]
$$

$E_{7}$ is a stable point if $a r_{2}\left(\theta_{2} b K-d_{2}\right)>\theta_{2} b^{2} K\left(\lambda_{0} K-d_{1}\right)$. Note that this condition coincides with $\mathcal{R}_{1}<1$. Otherwise it is a saddle with a one-dimensional unstable manifold.

For $E_{8}$, one eigenvalue is $r_{2}-b P_{8}$ and others are the characteristic roots of the matrix,

$$
M=\left[\begin{array}{ccc}
-\frac{r_{1} S_{8}}{K} & -\frac{r_{1} S_{8}}{K}-\lambda_{0} S_{8} & 0 \\
\lambda_{0} I_{8} & 0 & -a I_{8} \\
0 & P_{8} \theta_{1} a & 0
\end{array}\right]
$$

If we write the characteristic equation for $M_{8}$, we get a cubic equation,

$$
\mu^{3}+B_{1} \mu^{2}+B_{2} \mu+B_{3}=0
$$

and its coefficients satisfy the conditions $B_{1}>0, B_{3}>0$ and $B_{1} B_{2}>B_{3}$. Hence, $E_{8}$ is locally asymptotically stable if $r_{2}<b P_{8}$. Otherwise it is a saddle point with a one-dimensional unstable manifold.

Note 3.3. It is important to note here that the existence of the interior equilibrium requires that all boundary equilibria are unstable.

We shall prove the local stability of the interior equilibrium point with the help of a suitable Lyapunov function. Linearizing the system (3.1) - (3.4) around the interior equilibrium point with help of the 


\begin{tabular}{|c|c|c|}
\hline Equilibrium & Existence & Stability \\
\hline$E_{0}(0,0,0,0)$ & Always & $\begin{array}{l}\text { Saddle point with two-dimensional stable } \\
\text { and two-dimensional unstable manifolds. }\end{array}$ \\
\hline$E_{1}(K, 0,0,0)$ & Always & $\begin{array}{l}\text { Saddle point, dimension of unstable manifold } \\
\text { is two if } \boldsymbol{H}_{\mathbf{1}}\left(\boldsymbol{R}_{\mathbf{0}}<\mathbf{1}\right) \text { and one otherwise }\end{array}$ \\
\hline$E_{2}(0,0, K, 0)$ & Always & $\begin{array}{l}\text { Saddle point, dimension of unstable manifold } \\
\text { is two if } \boldsymbol{H}_{\mathbf{2}} \text { holds and one otherwise. }\end{array}$ \\
\hline$E_{3}(K, 0, K, 0)$ & Always & $\begin{array}{c}\text { Locally asymptotically stable if both } \boldsymbol{H}_{1}\left(\mathcal{R}_{0}<1\right) \text { and } \boldsymbol{H}_{2} \\
\text { do not hold, otherwise it is a saddle point }\end{array}$ \\
\hline$E_{4}\left(S_{4}, I_{4}, 0,0\right)$ & If $\boldsymbol{H}_{1}$ holds. & $\begin{array}{l}\text { Saddle point, dimension of unstable manifold } \\
\text { is two if } \boldsymbol{H}_{4} \text { holds and one otherwise. }\end{array}$ \\
\hline$E_{5}\left(0,0, N_{5}, P_{5}\right)$ & If $\boldsymbol{H}_{2}$ holds. & Saddle point, with one-dimensional unstable manifold. \\
\hline$E_{6}\left(S_{6}, I_{6}, K, 0\right)$ & If $\boldsymbol{H}_{1}$ holds. & $\begin{array}{l}\text { Locally asymptotically stable if } \boldsymbol{H}_{\mathbf{5}} \text { does not hold, } \\
\text { otherwise saddle point with one-dimensional unstable manifold. }\end{array}$ \\
\hline$E_{7}\left(K, 0, N_{7}, P_{7}\right)$ & If $\boldsymbol{H}_{2}$ holds. & $\begin{array}{l}\text { Locally asymptotically stable if } \boldsymbol{H}_{\mathbf{6}} \text { does not hold }\left(\boldsymbol{\mathcal { R }}_{1}<\mathbf{1}\right) \text {, } \\
\text { otherwise saddle point with one-dimensional unstable manifold }\end{array}$ \\
\hline$E_{8}\left(S_{8}, I_{8}, 0, P_{8}\right)$ & If both $\boldsymbol{H}_{3}$ and $\boldsymbol{H}_{4}$ hold. & $\begin{array}{l}\text { Locally asymptotically stable if } \boldsymbol{H}_{\boldsymbol{7}} \text { does not hold, } \\
\text { otherwise saddle point with one-dimensional unstable manifold }\end{array}$ \\
\hline$E_{*}\left(S_{*}, I_{*}, N_{*}, P_{*}\right)$ & If $\boldsymbol{H}_{5}, \boldsymbol{H}_{6}$ and $\boldsymbol{H}_{7}$ hold. & Locally asymptotically stable. \\
\hline
\end{tabular}

TABLE 2. Summary of existence and stability conditions for the equilibria of Model 1.

Note that $S_{4}=S_{6}, I_{4}=I_{6}, N_{5}=N_{7}$ and $P_{5}=P_{7}$.

transformation $S=S_{*}+x_{1}, I=I_{*}+x_{2}, N=N_{*}+x_{3}$ and $P=P_{*}+x_{4}$ we get the following linearized system,

$$
\begin{aligned}
& \dot{x}_{1}=-\alpha_{1} x_{1}-\alpha_{2} x_{2}, \\
& \dot{x}_{2}=\alpha_{3} x_{1}-\alpha_{4} x_{4}, \\
& \dot{x}_{3}=-\alpha_{5} x_{3}-\alpha_{6} x_{4}, \\
& \dot{x}_{4}=\alpha_{7} x_{2}+\alpha_{8} x_{3},
\end{aligned}
$$

where all $\alpha_{1}=\frac{r_{1} S_{*}}{K}, \alpha_{2}=\left(\frac{r_{1}}{K}+\lambda_{0}\right) S_{*}, \alpha_{3}=\lambda_{0} I_{*}=\alpha_{4}, \alpha_{5}=\frac{r_{2} N_{*}}{K}, \alpha_{6}=b N_{*}, \alpha_{7}=\theta_{1} a P_{*}$ and $\alpha_{8}=\theta_{2} b P_{*}$ are positive.

We can construct a Lyapunov function,

$$
V\left(x_{1}, x_{2}, x_{3}, x_{4}\right)=\frac{1}{2}\left(C_{1} x_{1}^{2}+C_{2} x_{2}^{2}+C_{3} x_{3}^{2}+C_{4} x_{4}^{2}\right),
$$

and then calculating the time derivative along the solution trajectories of the above linearized system, we get

$$
\frac{d V}{d t}=-C_{1} \alpha_{1} x_{1}^{2}-C_{3} \alpha_{5} x_{3}^{2}+\left(C_{3} \alpha_{3}-C_{1} \alpha_{2}\right) x_{1} x_{2}+\left(C_{4} \alpha_{7}-C_{2} \alpha_{4}\right) x_{2} x_{4}+\left(C_{4} \alpha_{8}-C_{3} \alpha_{6}\right) x_{3} x_{4} .
$$

Choosing, $C_{1}=1, C_{2}=\alpha_{2} \alpha_{6} \alpha_{7} / \alpha_{3} \alpha_{4} \alpha_{8}>0, C_{3}=\alpha_{2} / \alpha_{3}>0$ and $C_{4}=\alpha_{2} \alpha_{6} / \alpha_{3} \alpha_{8}>0$, we find

$$
\frac{d V}{d t}=-\alpha_{1} x_{1}^{2}-C_{3} \alpha_{5} x_{3}^{2} \leq 0 .
$$

Hence, the interior equilibrium $\left(S_{*}, I_{*}, N_{*}, P_{*}\right)$ is always locally stable whenever this equilibrium exists.

The main results on the stability of the equilibria in Model 1 are summarized in Table 2. 


\subsection{Bifurcation Portraits of Model 1}

We follow changes in the species equilibrium densities by considering the transmission rate coefficient $\lambda_{0}$ as the key bifurcation parameter. The outcomes largely depend on whether or not the alternative prey can support the predator's growth in the absence of disease in the first prey.

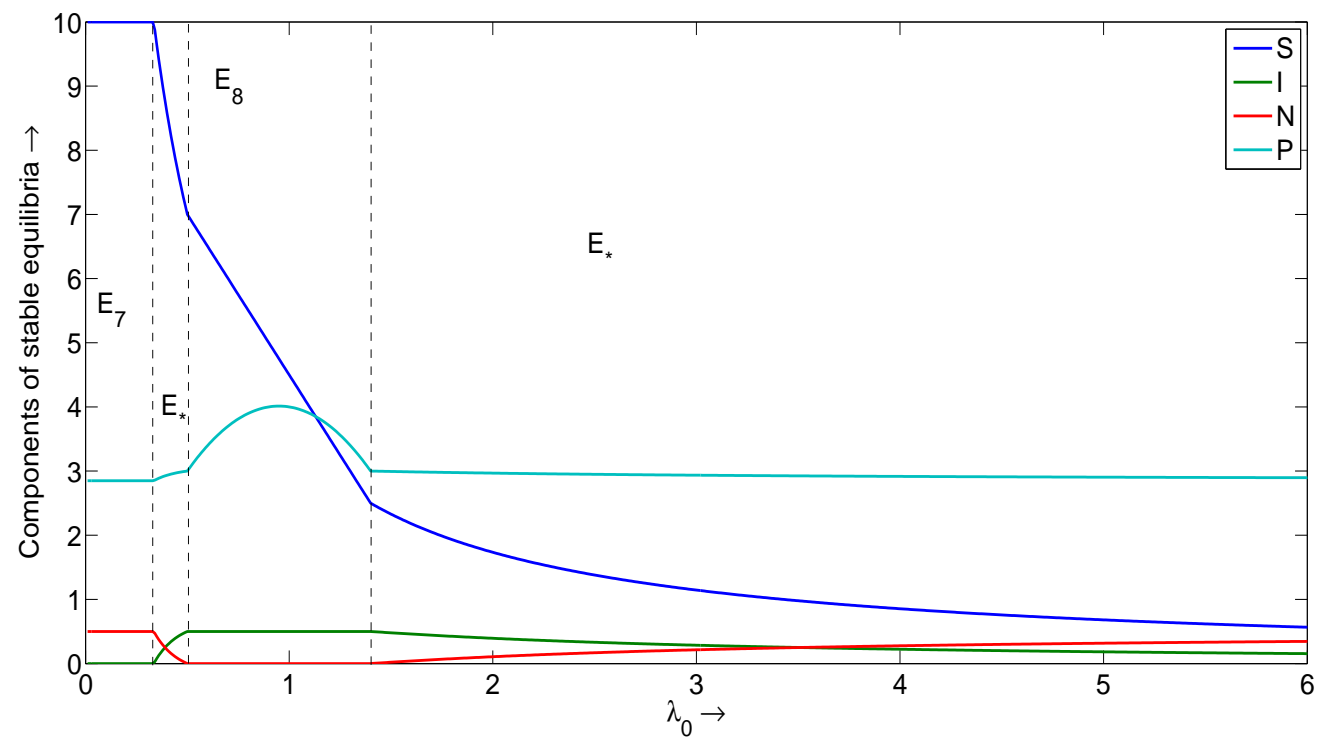

Figure 2. Equilibrium species densities in Model 1 are constructed as functions of the transmission coefficient $\lambda_{0}$. The other model parameters are $r_{1}=1, K=10, d_{1}=.5$, $a=1, r_{2}=3, b=1, \theta_{1}=.2, \theta_{2}=.2, d_{2}=.1$

In the case, where prey \#2 can alone support the predator $\left(\boldsymbol{H}_{\mathbf{2}}\right.$ is satisfied), a typical bifurcation structure is represented in Fig.2 (constructed for $r_{1}=1, K=10, d_{1}=.5, a=1, r_{2}=3, b=1$, $\left.\theta_{1}=.2, \theta_{2}=.2, d_{2}=.1\right)$. The figure shows the stable equilibria, which are the only model attractors of the system. For a weak transmission rate, the disease cannot establish in the system and the entire population of prey \#1 is healthy. Starting from a critical value of $\lambda_{0}$ (determined by the condition $\mathcal{R}_{1}=1$ ), the disease invades the system and all four components $S, I, N, P$ can coexist (equilibrium is $E_{*}$ ). Interestingly, for a further increase of disease transmission, the density of prey \#2 tends to zero and this species goes extinct (equilibrium $E_{8}$ ). This can be explained by the fact that the predator density becomes large and the predation rate on prey \#2 becomes higher than its maximal growth rate $r_{2}$. A further gradual increase in $\lambda_{0}$ results in a drop of $I$, and, as a consequence, a decrease in $P$, thus the predation on prey $\# 2$ is reduced and it can establish itself in the system again (equilibrium $E_{*}$ ). For a further increase in the transmission rate, the values of $S, I, P$ decrease, whereas that of $N$ slightly increases. It is worth mentioning that in Fig.2 and other bifurcation diagrams, the exchange of stability among the equilibria takes place through transcritical bifurcations.

The extinction of $N$ at some intermediate $\lambda_{0}$ requires relatively high values of the carrying capacity and does not occur at low $K$, although the values of $N$ generally drop. For instance, if we drop $K$ from 10 to 2 , the stationary value of $N$ is always positive (the diagram is not shown here).

In the case where prey \#2 cannot support the growth of predator alone, the bifurcation diagrams radically change. Fig. 3 shows an example of such a situation (we decreased the value of the conversion coefficient $\theta_{2}$, and the carrying capacity $K$, the other parameters are the same as before). One can 
see from the diagram that the predator can survive only within an intermediate range of $\lambda_{0}$ : for small transmission rate the disease cannot establish in the system, thus the predator cannot consume infected prey \#1; for high transmission rates the level of infected prey $I$ becomes small and it cannot support the predator growth. In this sense, the diagram is somewhat similar to that of Fig.1 (i.e. in the will be observed for larger values of $K$ (not shown here): in this case an increase in $P$ for some intermediate transmission rate is high enough to completely suppress the growth of prey \#2. This is an example of strong apparent competition of the prey species via their common predator [32].

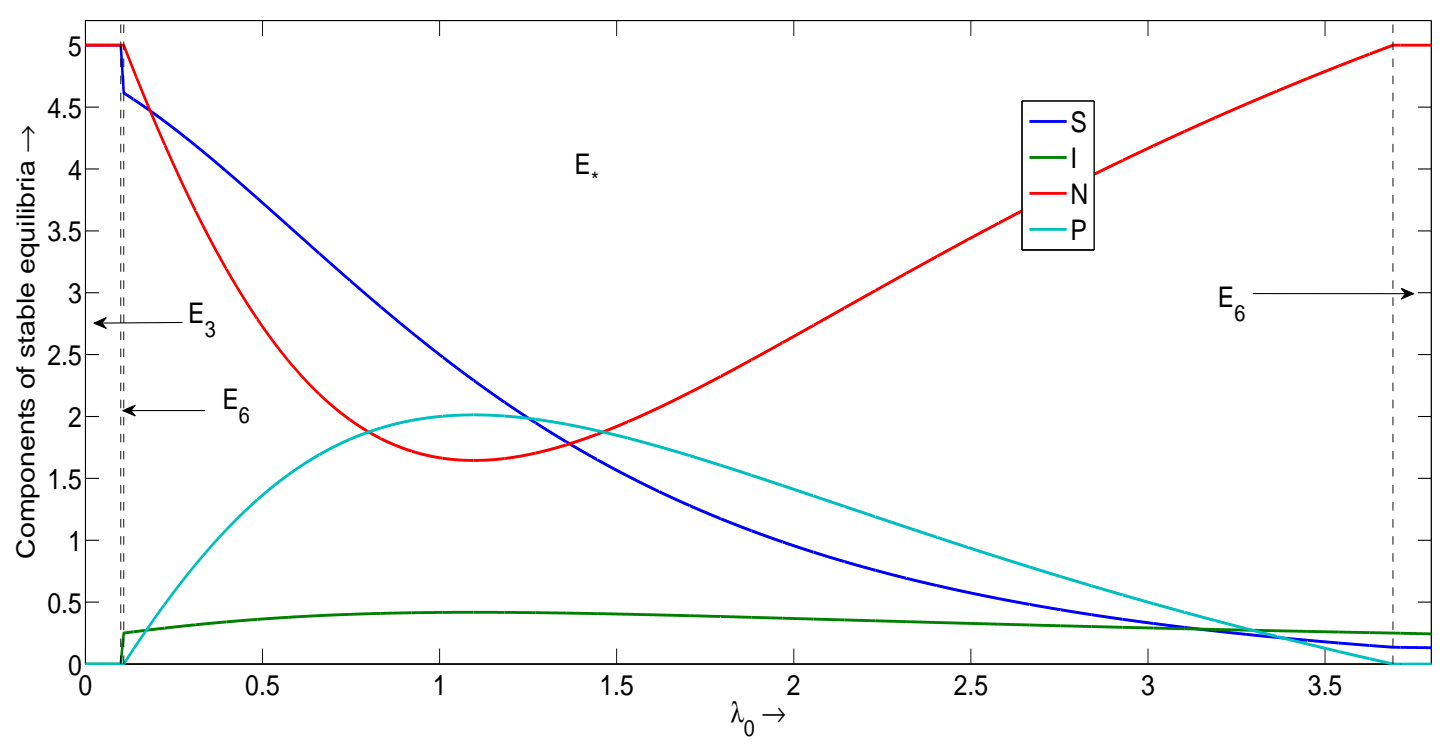

FiguRE 3. Equilibrium species densities in Model 1 are constructed as functions of the transmission coefficient $\lambda_{0}$. The other model parameters are $r_{1}=1, K=5, d_{1}=.5$, $a=1, r_{2}=3, b=1, \theta_{1}=.2, \theta_{2}=.01, d_{2}=.1$.

Interestingly, the presence of an alternative prey can strongly affect the disease prevalence, which is an important characteristic of the infection strength [28]. The prevalence is determined as the ratio between the infected individuals and the total number of individuals in the population, i.e. $I /(I+S)$. Fig.4 shows the graphs of the dependence of the prevalence on $\lambda_{0}$ in the presence (solid line) and in the absence (dashed line) of the second predator. One can see that for $N>0$, the disease prevalence exhibits a substantial drop. This is due to the fact that in the presence of prey \#2, the population size of the healthy prey \#1, S, increases. Such a decrease in the disease prevalence is observed within a broad range of model parameters.

Finally, we also investigated the dependence of the system behaviour on both the transmission rate $\lambda_{0}$ and the virulence $d_{1}$. Fig.5 shows two different examples of $\lambda_{0}-d_{1}$ diagrams constructed for the same parameters as in Fig.2 and Fig. 3 respectively. The symbols $E_{i}$ denote the stable equilibria of the system (see Table 2), which are the global model attractors. In the case where the second prey can alone support the growth of the predator (Fig.5a), an increase in virulence impedes the disease establishment (the area corresponding to $E_{7}$ becomes larger); moreover, for high $d_{1}$, extinction of the second prey is not observed due to the fact that the density of the predator drops. In the situation where the second prey does not provide enough supply for the predator (see Fig.5b), the persistence of predator is possible only for small and intermediate $d_{1}$ : for large values of virulence, the predator cannot survive due to a low level of $I$. 


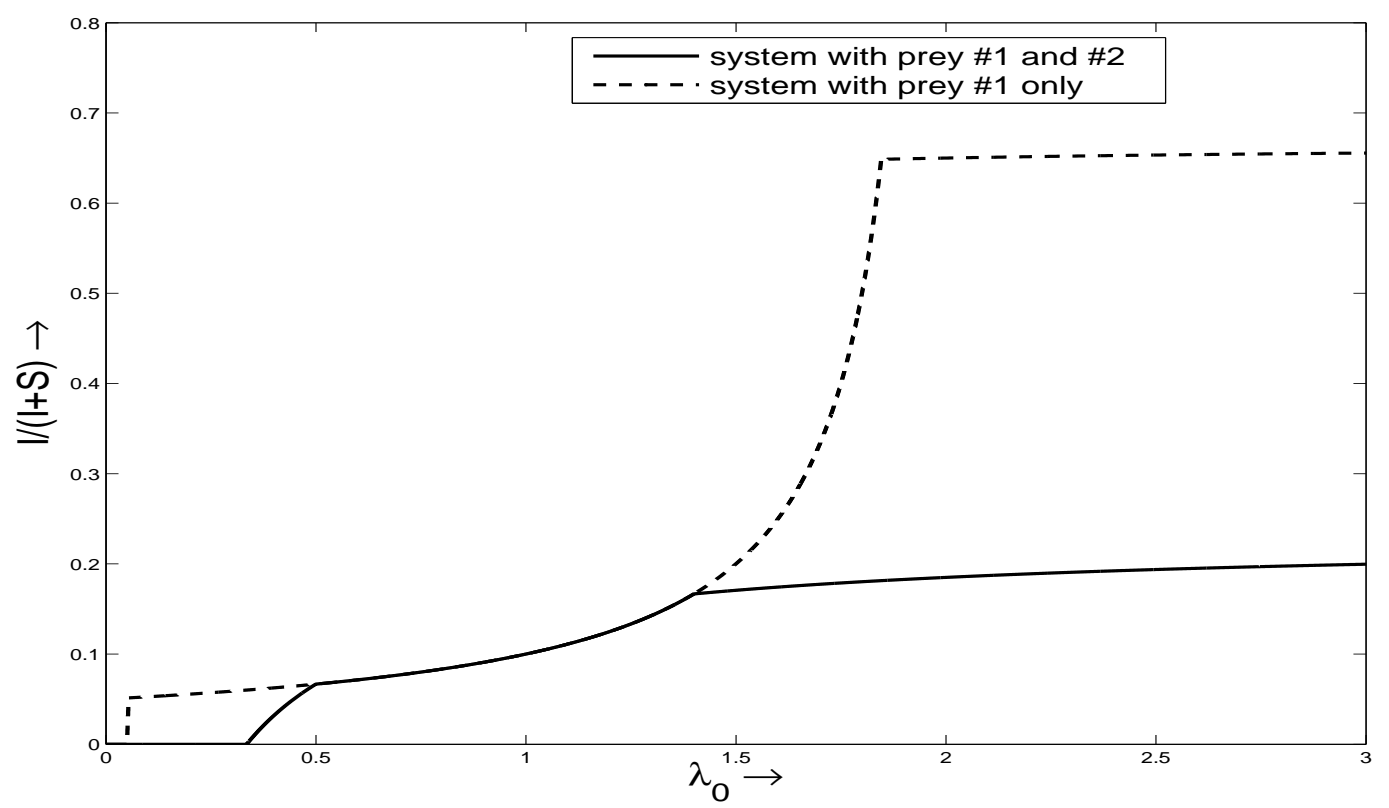

Figure 4. The disease prevalence in the system with two prey species (solid line) and in the system with a single prey (dashed line). The model parameters are the same as in Fig.2

\section{Model 2: predator-dependent disease transmission scenario}

For a predator-dependent transmission scenario $(\alpha>0)$, the model equations read

$$
\begin{aligned}
\frac{d S}{d t} & =r_{1}\left(1-\frac{S+I}{K}\right) S-\left(\lambda_{0}+\alpha P\right) S I \\
\frac{d I}{d t} & =\left(\lambda_{0}+\alpha P\right) S I-d_{1} I-a I P \\
\frac{d N}{d t} & =r_{2}\left(1-\frac{N}{K}\right) N-b N P \\
\frac{d P}{d t} & =\theta_{1} a I P+\theta_{2} b N P-d_{2} P
\end{aligned}
$$

We shall start our analysis with a brief description of the model behaviour in the absence of prey \#2, since this will be important for understanding the consequences of introducing the alternative prey. The model has been considered in detail in [25] and we briefly list the main findings.

In the absence of prey $\# 2$, the model has the following equilibria. The trivial stationary equilibrium is $G_{0}=(0,0,0)$; it is always unstable. The disease-free and predator-free equilibrium is given by $G_{1}=$ $(K, 0,0)$; it is stable for $D>K \lambda_{0}$ and unstable otherwise. The predator-free stationary equilibrium is given by $G_{2}=\left(S_{6}, I_{6}, 0\right)$, with $S_{6}=d_{1} / \lambda, I_{6}=r\left(K \lambda_{0}-d_{1}\right) /\left(\lambda_{0}\left(K \lambda_{0}+r\right)\right)$. Finally, there is an interior stationary state $\left(G_{*}=S_{8(1,2)}, I_{8(1,2)}, P_{8(1,2)}\right)$, where the stationary densities are determined by

$$
S_{8(1,2)}=\frac{d_{1}+a P_{8(1,2)}}{\lambda_{0}+\alpha P_{8(1,2)}}, I_{8(1,2)}=\frac{\delta}{\theta_{1} a},
$$




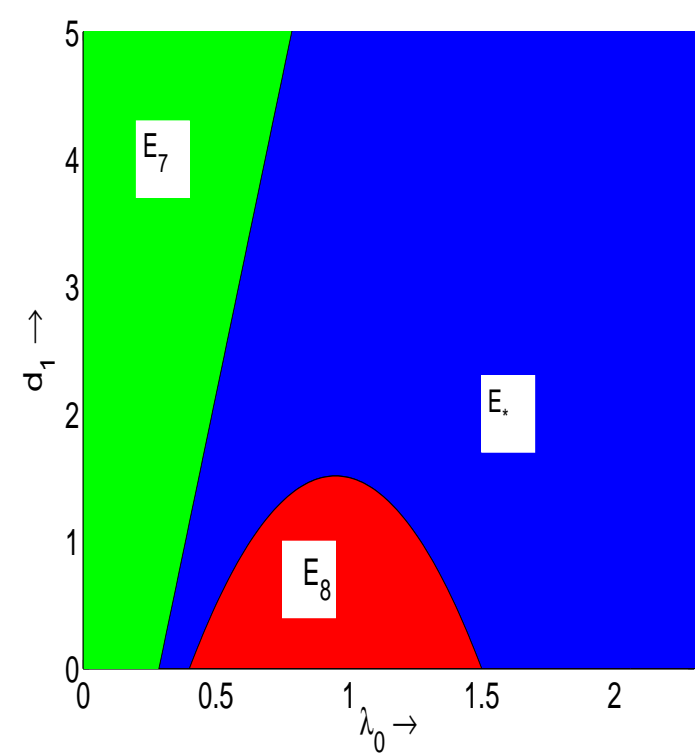

(a)

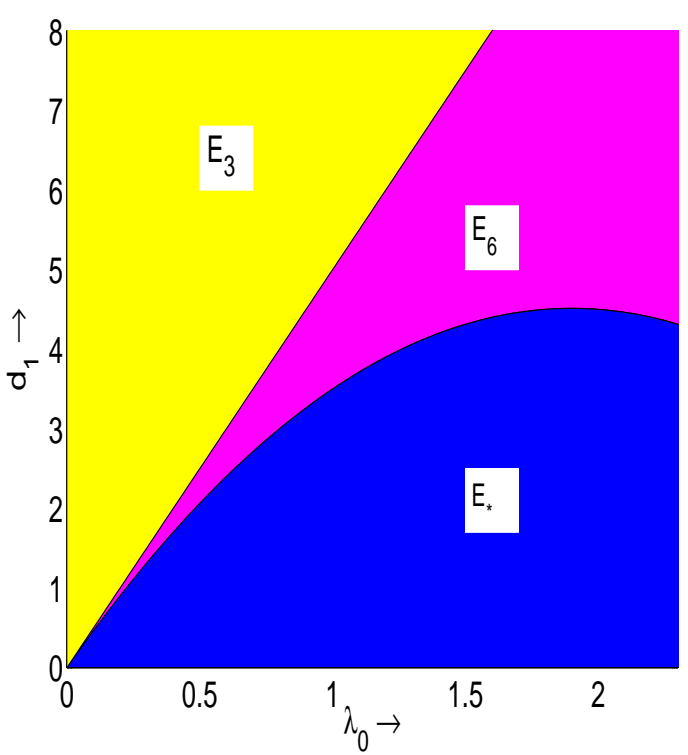

(b)

Figure 5. Bifurcation diagram of Model 1 in the $\lambda_{0}-d_{1}$-plane for constructed for: $r_{1}=1, K=10, a=1, r_{2}=3, b=1, \theta_{1}=.2, \theta_{2}=.2, d_{2}=.1$ (left panel); $r_{1}=1$, $K=5, a=1, r_{2}=3, b=1, \theta_{1}=.2, \theta_{2}=.01, d_{2}=.1$ (right panel).

where $P_{8(1,2)}$ is one of the positive roots of the following quadratic equation,

$$
D_{0} P_{8(1,2)}^{2}+D_{1} P_{8(1,2)}+D_{2}=0
$$

with

$$
\begin{aligned}
& D_{0}=\alpha^{2} d_{2} K, \\
& D_{1}=2 \alpha d_{2} K \lambda_{0}+\alpha d_{2} r_{1}+a^{2} r_{1} \theta_{1}-a \alpha K r_{1} \theta_{1}, \\
& D_{2}=d_{2} \lambda_{0}\left(r_{1}+\lambda_{0} K\right)-\theta_{1} a r_{1}\left(\lambda_{0} K-d_{1}\right) .
\end{aligned}
$$

If $\boldsymbol{H}_{\mathbf{8}}: D_{1}<0$ and $D_{1}^{2}>4 D_{0} D_{2}$ hold, the system has two interior equilibria $\left(S_{8(1,2)}, I_{8(1,2)}, P_{8(1,2)}\right)$, one of them is a always saddle point. Note that condition $\boldsymbol{H}_{\mathbf{4}}\left(D_{2}<0\right)$ is also required in this case.

Adding predator-dependent disease transmission (compared to the same model with $\alpha=0$ ) results in the possibility of multiple attractors [25], which was impossible for $\alpha=0$. A typical bifurcation diagram in the $\lambda_{0}-\alpha$ plane is shown in Fig.6, which is constructed for the same parameters as Fig.1. The labels over the domains indicate the attractors of the system. We also show close-ups of two parts of the diagram with a more complex structure (see Fig.6, the bottom panels). From the diagrams one can see that for a small $\alpha$, a gradual increase in the predator-independent transmission $\lambda_{0}$ results in the same behaviour as for $\alpha=0$ (cf. Fig.1). However, for intermediate $\alpha$ the system exhibits bi-stability for small $\lambda_{0}$ : depending on initial condition, the predator will either establish in the system or go extinct. Finally, for some large $\alpha$, the interior equilibrium becomes unstable and the species densities periodically oscillate around this equilibrium. A further increase in the predator-independent transmission component $\lambda_{0}$ (for a fixed $\alpha$ ) results in qualitatively similar behaviour to the case $\alpha=0$. Thus, the main alteration caused by a predator-dependent disease transmission is observed only for small $\lambda_{0}$ (see [25] for more detail).

Having the above properties in mind, we shall proceed to the investigation of the full Model 2. 


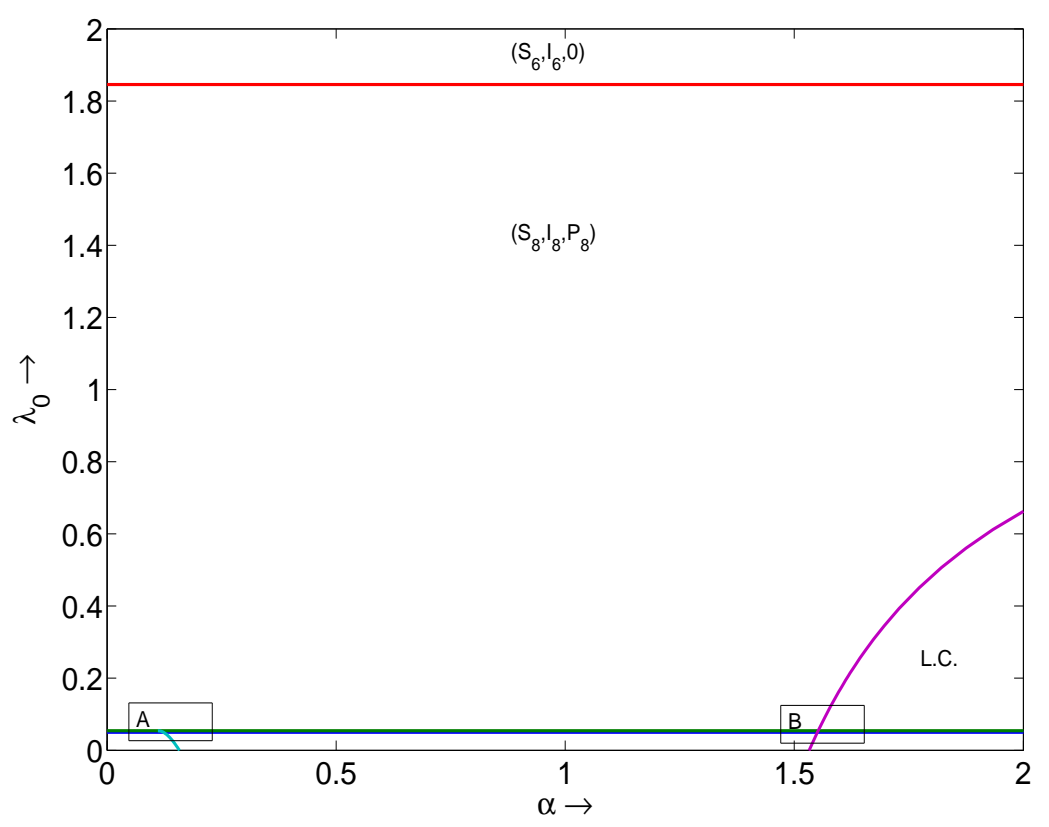

A

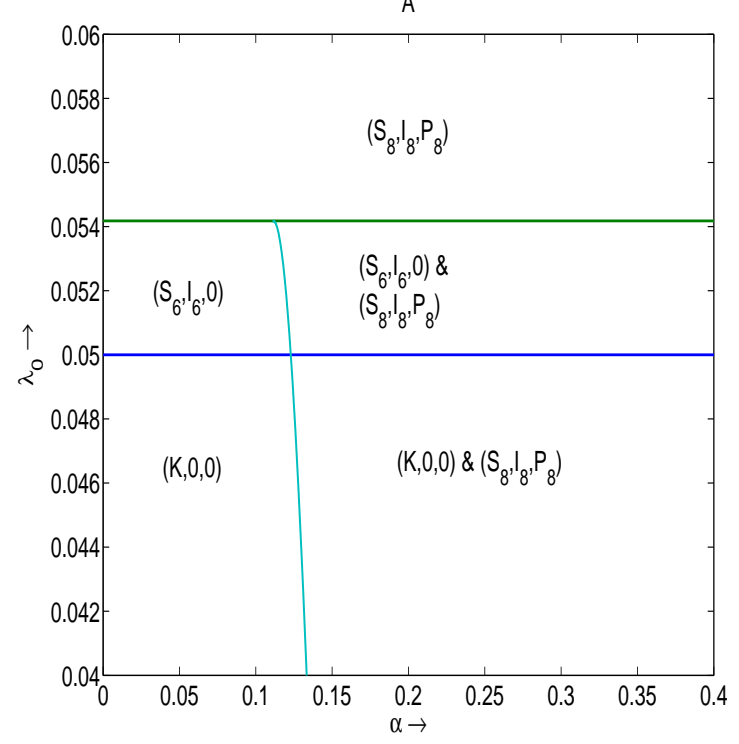

B

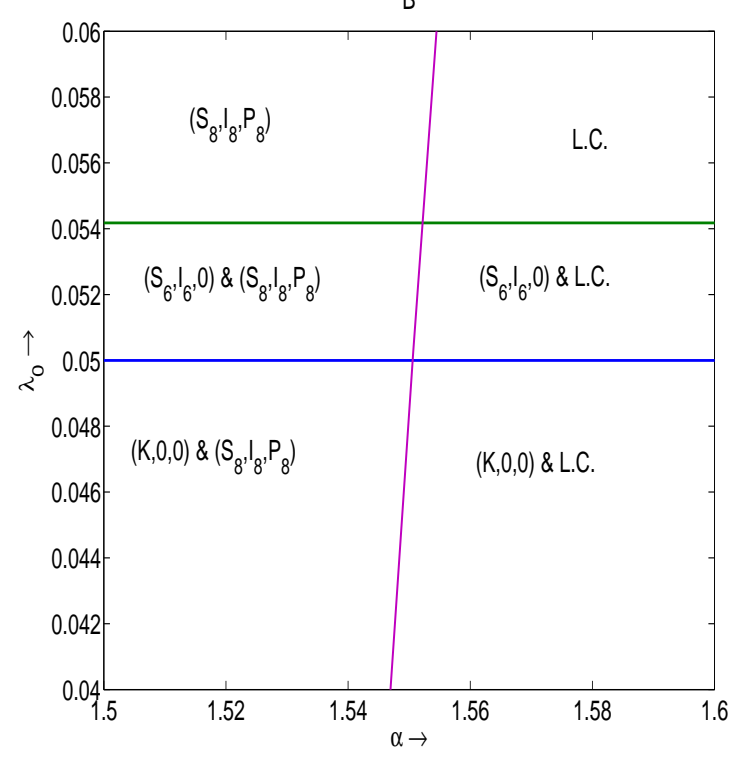

Figure 6 . Bifurcation diagram in $\alpha-\lambda_{0}$-plane for Model 2 in the absence of prey \#2. The other parameters are: $r_{1}=1, K=10, a=1, d_{1}=.5, \theta_{1}=.2, d_{2}=.1$. The symbols $G_{i}$ denote the corresponding attracting equilibria $\left(G_{i}\right)$ and a stable limit cycle (L.C.).

\subsection{Equilibria}

The full model (4.1)-(4.4) has the following equilibria: the trivial equilibrium point $E_{0}(0,0,0,0)$, two axial equilibria $E_{1}(K, 0,0,0), E_{2}(0,0, K, 0)$ and one boundary equilibrium point given by $E_{3}(K, 0, K, 0)$. 
These equilibria always exist. As it was the case with Model 1, Model 2 will have four boundary equilibria given by $E_{4}\left(\frac{d_{1}}{\lambda_{0}}, \frac{r_{1}\left(\lambda_{0} K-d_{1}\right)}{\lambda_{0}\left(r_{1}+\lambda_{0} K\right)}, 0,0\right), E_{5}\left(0,0, \frac{r_{2}\left(\theta_{2} b K-d_{2}\right)}{\theta_{2} b^{2} K}, \frac{d_{2}}{\theta_{2} b}\right), E_{6}\left(\frac{d_{1}}{\lambda_{0}}, \frac{r_{1}\left(\lambda_{0} K-d_{1}\right)}{\lambda_{0}\left(r_{1}+\lambda_{0} K\right)}, K, 0\right)$, $E_{7}\left(K, 0, \frac{d_{2}}{\theta_{2} b}, \frac{r_{2}\left(\theta_{2} b K-d_{2}\right)}{\theta_{2} b^{2} K}\right)$ under the parametric restrictions stated in Proposition 3.1.

On the other hand, the model can have the following two boundary equilibria of the form $E_{8 i}\left(\frac{d_{1}+a P_{8}(i)}{\lambda_{0}+\alpha P_{8}(i)}, \frac{d_{2}}{\theta_{1} a}, 0, P_{8}(i)\right)$, where $P_{8}(i)$ is a positive solution of the equation (4.6). In this case the second prey is absent and the equilibrium densities coincide with those of (4.6)- (4.5). Computation of the stationary densities of the predator gives $P_{8}(1)=\frac{-D_{1}-\sqrt{D_{1}^{2}-4 D_{0} D_{2}}}{2 D_{0}}$ and $P_{8}(2)=\frac{-D_{1}+\sqrt{D_{1}^{2}-4 D_{0} D_{2}}}{2 D_{0}}$, where the values of $D_{i}$ are provided by (4.7) - (4.9). Hence, if $\boldsymbol{H}_{4}$ : holds only $E_{82}$ exists. Otherwise both $E_{81}$ and $E_{82}$ are feasible provided $\boldsymbol{H}_{\mathbf{8}}: D_{1}<0$ and $D_{1}^{2}>4 D_{0} D_{2}$ hold.

Finally, Model 2 may have up to three interior equilibrium points depending upon the parametric restrictions. Let us denote an interior equilibrium point by $E_{*}\left(S_{*}, I_{*}, N_{*}, P_{*}\right)$, where $I_{*}$ is a positive root of the cubic equation

$$
\Delta_{0} \xi^{3}+\Delta_{1} \xi^{2}+\Delta_{2} \xi+\Delta_{3}=0
$$

where

$$
\begin{aligned}
\Delta_{0}= & \alpha^{2} r_{2}^{2} \theta_{1}^{2} a^{2} \\
\Delta_{1}= & \left(2 \alpha^{2} r_{2}^{2} \theta_{1} a \theta_{2} b K+2 r_{2} \alpha K \lambda_{0} \theta_{2} b^{2} \theta_{1} a+r_{2} \alpha r_{1} \theta_{2} b^{2} \theta_{1} a-2 \alpha^{2} r_{2}^{2} \theta_{1} a d_{2}\right), \\
\Delta_{2}= & \left(r_{2} a^{2} r_{1} \theta_{2} b^{2} \theta_{1}-r_{2} \alpha r_{1} \theta_{2} b^{2} d_{2}+r_{2} \alpha r_{1} \theta_{2}^{2} b^{3} K-r_{2} \alpha r_{1} K \theta_{2} b^{2} \theta_{1} a+\lambda_{0} r_{1} \theta_{2}^{2} b^{4} K+2 r_{2} \alpha K^{2} \lambda_{0} \theta_{2}^{2} b^{3}\right. \\
& \left.+\alpha^{2} r_{2}^{2} \theta_{2}^{2} b^{2} K^{2}-2 \alpha^{2} r_{2}^{2} \theta_{2} b K d_{2}+\alpha^{2} r_{2}^{2} d_{2}^{2}+K^{2} \lambda_{0}^{2} \theta_{2}^{2} b^{4}-2 r_{2} \alpha K \lambda_{0} \theta_{2} b^{2} d_{2}\right) \\
\Delta_{3}= & -r_{2} a r_{1} \theta_{2} b^{2} d_{2}-\lambda_{0} r_{1} K^{2} \theta_{2}^{2} b^{4}-r_{2} \alpha r_{1} K^{2} \theta_{2}^{2} b^{3}+r_{2} a r_{1} \theta_{2}^{2} b^{3} K+d_{1} r_{1} \theta_{2}^{2} b^{4} K+r_{2} \alpha r_{1} K \theta_{2} b^{2} d_{2} .
\end{aligned}
$$

Other components are given by

$$
\begin{gathered}
S_{*}=\frac{r_{1} K \theta_{2} b^{2}+\alpha r_{2} d_{2} I_{*}-r_{1} \theta_{2} b^{2} I_{*}+K \lambda_{0} \theta_{2} b^{2} I_{*}-\alpha a r_{2} \theta_{1} I_{*}-\alpha r_{2} \theta_{2} b K I_{*}}{r_{1} \theta_{2} b^{2}} \\
N_{*}=\frac{d_{2}-\theta_{1} a I_{*}}{\theta_{2} b}, \\
P_{*}=\frac{r_{2}\left(\theta_{1} a I_{*}+\theta_{2} b K-d_{2}\right)}{\theta_{2} b^{2} K} .
\end{gathered}
$$

We define the following condition:

$\boldsymbol{H}_{\mathbf{9}}$ : In the case the cubic equation (4.10) has one, two or three positive roots $I_{i *}$ such that $S_{i *}>$ $0, N_{i *}>0, P_{i *}>0$, for $i=1,2$ or 3 , the model will have one, two or three feasible interior equilibrium points.

The explicit expressions for $\boldsymbol{H}_{\mathbf{9}}$ : can be technically derived; however, obviously, they are rather cumbersome and we do not provide them here.

The main results on system equilibria in Model 1 are summarized in Table 3.

Note that adding a predator-dependent disease transmission $\alpha>0$ alters the previous condition $\mathcal{R}_{1}>1$ (obtained in Model 1) for the establishment of the disease in the presence of the second predator (see (3.7)). Thus, we need to introduce a modified basic reproduction number $\mathcal{R}_{2}$. We again consider the per capita growth rate of $I$ under condition that the predator density is supported by the second prey $\left(P=P_{7}\right)$ and proceed in a similar way as before. We define $\mathcal{R}_{2}$ as

$$
\mathcal{R}_{2}=\frac{\left(\lambda_{0}+\alpha P_{7}\right) K}{d_{1}+a P_{7}}=\frac{\left(\lambda_{0}+\alpha \frac{r_{2}\left(\theta_{2} b K-d_{2}\right)}{\theta_{2} b^{2} K}\right) K}{d_{1}+\frac{a r_{2}\left(\theta_{2} b K-d_{2}\right)}{\theta_{2} b^{2} K}} .
$$


Thus, a necessary condition for an endemic equilibrium $E_{*}$ is given by $\mathcal{R}_{2}>1$.

\subsection{Stability of equilibria}

The Jacobian matrix for the above model evaluated at an arbitrary point is given by

$$
J=\left[\begin{array}{cccc}
r_{1}\left(1-\frac{2 S+I}{K}\right)-\left(\lambda_{0}+\alpha P\right) I & -r_{1} \frac{S}{K}-\left(\lambda_{0}+\alpha P\right) S & 0 & -\alpha S I \\
\left(\lambda_{0}+\alpha P\right) I & \left(\lambda_{0}+\alpha P\right) S-d_{1}-a P & 0 & (\alpha S-a) I \\
0 & 0 & r_{2}\left(1-\frac{2 N}{K}\right)-b P & -b N \\
0 & \theta_{1} a P & \theta_{2} b P & \theta_{1} a I+\theta_{2} b N-d_{2}
\end{array}\right]
$$

Evaluating the Jacobian matrix at $E_{0}(0,0,0,0)$, the trivial equilibrium point, we find the eigenvalues of the Jacobian matrix as $r_{1}>0,-d_{1}<0, r_{2}>0,-d_{2}<0$, and hence $E_{0}$ is a saddle-point with a two-dimensional stable manifold and two dimensional unstable manifold.

The eigenvalues of the Jacobian matrix evaluated at $E_{1}(K, 0,0,0)$ are $-r_{1}<0, \lambda_{0} K-d_{1}, r_{2}>0$ and $-d_{2}<0$. Hence $E_{1}$ is a saddle point, the dimension of the unstable manifold is 2 if $\lambda_{0} K>d_{1}$ and is 1 otherwise.

Evaluating $J$ at $E_{2}(0,0, K, 0)$ we find the related eigenvalues $r_{1}>0,-d_{1}<0,-r_{2}<0$ and $\theta_{2} b K-d_{2}$. Hence $E_{2}$ is a saddle point having a two-dimensional unstable manifold if $\theta_{2} b K>d_{2}$.

Evaluating the Jacobian matrix at the first boundary equilibrium point $E_{3}(K, 0, K, 0)$ we find its related eigenvalues $-r_{1}<0, \lambda_{0} K-d_{1},-r_{2}<0$ and $\theta_{2} b K-d_{2}$. Hence $E_{3}$ is locally asymptotically stable if $K<\min \left\{\frac{d_{1}}{\lambda_{0}}, \frac{d_{2}}{\theta_{2} b}\right\} . \quad E_{3}$ is a saddle point having a two-dimensional unstable manifold if $K>\max \left\{\frac{d_{1}}{\lambda_{0}}, \frac{d_{2}}{\theta_{2} b}\right\}$

For $E_{4}\left(\frac{d_{1}}{\lambda_{0}}, \frac{r_{1}\left(\lambda_{0} K-d_{1}\right)}{\lambda_{0}\left(r_{1}+\lambda_{0} K\right)}, 0,0\right)$, the Jacobian matrix is

$$
J_{4}=\left[\begin{array}{cccc}
-\frac{r_{1} d_{1}}{\lambda_{0} K} & -\frac{r_{1} d_{1}}{\lambda_{0} K}-d_{1} & 0 & -\frac{r_{1} \alpha d_{1}\left(\lambda_{0} K-d_{1}\right)}{\lambda_{0}^{2}\left(r_{1}+\lambda_{0} K\right)} \\
\frac{r_{1}\left(\lambda_{0} K-d_{1}\right)}{r_{1}+\lambda_{0} K} & 0 & 0 & \frac{r_{1}\left(\alpha d_{1}-a \lambda_{0}\right)\left(\lambda_{0} K-d_{1}\right)}{\lambda_{0}^{2}\left(r_{1}+\lambda_{0} K\right)} \\
0 & 0 & r_{2} & 0 \\
0 & 0 & 0 & \frac{\theta_{1} a r_{1} \lambda_{0} K-\lambda_{0} d_{2} r_{1}-d_{2} \lambda_{0}^{2} K-d_{1} \theta_{1} r_{1} K}{\lambda_{0}\left(r_{1}+\lambda_{0} K\right)}
\end{array}\right]
$$

$E_{4}$ is a saddle point with a two-dimensional unstable manifold if $\theta_{1} a r_{1}\left(\lambda_{0} K-d_{1}\right)>d_{2} \lambda_{0}\left(r_{1}+\lambda_{0} K\right)$. Otherwise it will have a one-dimensional unstable manifold and a three-dimensional stable manifold.

For $E_{5}\left(0,0, \frac{r_{2}\left(\theta_{2} b K-d_{2}\right)}{\theta_{2} b^{2} K}, \frac{d_{2}}{\theta_{2} b}\right)$

$$
J_{5}=\left[\begin{array}{cccc}
r_{1} & 0 & 0 & 0 \\
0-\frac{b^{2} \theta_{2} K d_{1}+a r_{2}\left(\theta_{2} b K-d_{2}\right)}{\left(b^{2} \theta_{2} K\right)} & 0 & 0 \\
0 & 0 & -\frac{r_{2} d_{2}}{K \theta_{2} b} & -\frac{d_{2}}{\theta_{2}} \\
0 & \frac{\theta_{1} a r_{2}\left(\theta_{2} b K-d_{2}\right)}{b^{2} \theta_{2} K} & \frac{r_{2}\left(\theta_{2} b K-d_{2}\right)}{b K} & 0
\end{array}\right]
$$

$E_{5}$ is a saddle point with a one-dimensional unstable manifold.

For $E_{6}\left(\frac{d_{1}}{\lambda_{0}}, \frac{r_{1}\left(\lambda_{0} K-d_{1}\right)}{\lambda_{0}\left(r_{1}+\lambda_{0} K\right)}, K, 0\right)$

$$
J_{6}=\left[\begin{array}{cccc}
-\frac{r_{1} d_{1}}{\lambda_{0} K} & -\frac{r_{1} d_{1}}{\lambda_{0} K}-d_{1} & 0 & -\frac{r_{1} \alpha d_{1}\left(\lambda_{0} K-d_{1}\right)}{\lambda_{0}^{2}\left(r_{1}+\lambda_{0} K\right)} \\
\frac{r_{1}\left(\lambda_{0} K-d_{1}\right)}{r_{1}+\lambda_{0} K} & 0 & 0 & \frac{r_{1}\left(\alpha d_{1}-a \lambda_{0}\right)\left(\lambda_{0} K-d_{1}\right)}{\lambda_{0}^{2}\left(r_{1}+\lambda_{0} K\right)} \\
0 & 0 & -r_{2} & -b K \\
0 & 0 & 0 & \frac{\theta_{1} a r_{1}\left(\lambda_{0} K-d_{1}\right)+\lambda_{0}\left(r_{1}+\lambda_{0} K\right)\left(\theta_{2} b K-d_{2}\right)}{\lambda_{0}\left(r_{1}+\lambda_{0} K\right)}
\end{array}\right]
$$


$E_{6}$ is a stable point if $\theta_{1} a r_{1}\left(\lambda_{0} K-d_{1}\right)+\lambda_{0}\left(r_{1}+\lambda_{0} K\right)\left(\theta_{2} b K-d_{2}\right)<0$ otherwise it is saddle point with a one-dimensional unstable manifold.

For $E_{7}\left(K, 0, \frac{d_{2}}{\theta_{2} b}, \frac{r_{2}\left(\theta_{2} b K-d_{2}\right)}{\theta_{2} b^{2} K}\right)$

$$
J_{7}=\left[\begin{array}{cccc}
-r_{1} & -r_{1}-\left(\lambda_{0}+\frac{\alpha r_{2}\left(\theta_{2} b K-d_{2}\right)}{b^{2} \theta_{2} K}\right) K & 0 & 0 \\
0 & \left(\lambda_{0}+\frac{\alpha r_{2}\left(\theta_{2} b K-d_{2}\right)}{b^{2} \theta_{2} K}\right) K-d_{1}-\frac{a r_{2}\left(\theta_{2} b K-d_{2}\right)}{b^{2} \theta_{2} K} & 0 & 0 \\
0 & 0 & -\frac{r_{2} d_{2}}{K \theta_{2} b} & -\frac{d_{2}}{\theta_{2}} \\
0 & \frac{\theta_{1} a r_{2}\left(\theta_{2} b K-d_{2}\right)}{b^{2} \theta_{2} K} & \frac{r_{2}\left(\theta_{2} b K-d_{2}\right)}{b K} & 0
\end{array}\right]
$$

$E_{7}$ is a stable point if $\boldsymbol{H}_{\mathbf{1 0}}:\left(\lambda_{0}+\frac{\alpha r_{2}\left(\theta_{2} b K-d_{2}\right)}{b^{2} \theta_{2} K}\right) K<d_{1}+\frac{a r_{2}\left(\theta_{2} b K-d_{2}\right)}{b^{2} \theta_{2} K}$, otherwise it is a saddle with a one-dimensional unstable manifold. Note that the condition $\boldsymbol{H}_{\mathbf{1 0}}$ coincides with the requirement for the modified basic reproduction number $\mathcal{R}_{2}<1$.

For $E_{8 i}$ we have,

$$
J_{8 i}=\left[\begin{array}{cccc}
-\frac{r_{1} S_{8 i}}{K} & -r_{1} \frac{S_{8 i}}{K}-\left(\lambda_{0}+\alpha P_{8 i}\right) S_{8 i} & 0 & -\alpha S_{8 i} I_{8 i} \\
\left(\lambda_{0}+\alpha P_{8 i}\right) I_{8 i} & 0 & 0 & \left(\alpha S_{8 i}-a\right) I_{8 i} \\
0 & 0 & r_{2}-b P_{8 i} & 0 \\
0 & \theta_{1} a P_{8 i} & \theta_{2} b P_{8 i} & 0
\end{array}\right] .
$$

Finally, for the interior equilibria $E_{i *}$ we have

$$
J=\left[\begin{array}{cccc}
-\frac{r_{1} S_{i *}}{K} & -r_{1} \frac{S_{i *}}{K}-\left(\lambda_{0}+\alpha P_{i *}\right) S_{i *} & 0 & -\alpha S_{i *} I_{i *} \\
\left(\lambda_{0}+\alpha P_{i *}\right) I_{i *} & 0 & 0 & \left(\alpha S_{i *}-a\right) I_{i *} \\
0 & 0 & 0 & -b N_{i *} \\
0 & \theta_{1} a P_{i *} & \theta_{2} b P_{i *} & 0
\end{array}\right] .
$$

Computation of the explicit characteristic equation results in a cumbersome expression, which can be done only numerically.

The main results on the stability of the equilibria in Model 1 are summarized in Table 3.

\subsection{Bifurcation Portraits of Model 2}

Note that it is rather hard to fulfil an exhaustive parametric analysis of Model 2 due to the large number of parameters and a dramatic increase in complexity caused by $\lambda(P)$. Here, to gain an introductory insight, we shall investigate the role of the second prey in the dynamics of the system in two major ecologically relevant scenarios. The two cases we shall consider are: (i) the second prey $N$ can alone support the predator in the absence of the first prey, and (ii) the infected population $I$ of the first prey is required for the persistence of the predator. Following [25], we shall use $\alpha$ and $\lambda_{0}$ as the key bifurcation parameters.

Fig.7 shows a typical $\alpha-\lambda_{0}$ bifurcation diagram in the case the predator can survive in the absence of prey \#1. The diagram is constructed for the same parameters as in Fig.2. The symbols over the different domains specify model attractors; they are always stable equilibria $E_{i}$ for the given parameter range.

Comparison of Fig.6 and Fig.7 shows that including prey \#2 suppresses oscillations, stabilizes the system and restricts the conditions for epidemic spread (determined by $\mathcal{R}_{2}>1$ ). On the other hand, the domains with multiple stability disappear from the parametric portrait: now for any small initial amount of $P$, the predator is able to establish in the system (see domain $E_{8}$ in Fig.7), which was not the case in the system with a specialist predator [25]. Interestingly, this happens even in the case where the second prey cannot ultimately establish itself in the system. Thus, even if for large times we have extinction of prey \#2, introducing any small amount of $N$ will make the equilibrium with $P=0$ unstable. Thus, no Allee-like effect phenomena are observed as was the case in Fig.6. For high values of $\alpha$, all four components $S, I, N, P$ will always establish in the system. Computation of the disease prevalence also 


\begin{tabular}{|c|c|c|}
\hline Equilibrium & Existence & Stability \\
\hline$E_{0}(0,0,0,0)$ & Always & $\begin{array}{l}\text { Saddle point with two-dimensional stable } \\
\text { and two-dimensional unstable manifolds. }\end{array}$ \\
\hline$E_{1}(K, 0,0,0)$ & Always & $\begin{array}{l}\text { Saddle point, dimension of unstable manifold } \\
\text { is two if } \boldsymbol{H}_{\mathbf{1}}\left(\boldsymbol{\mathcal { R }}_{\mathbf{0}}<\mathbf{1}\right) \text { and one otherwise }\end{array}$ \\
\hline$E_{3}(K, 0, K, 0)$ & Always & $\begin{array}{c}\text { Locally asymptotically stable if both } \boldsymbol{H}_{\mathbf{1}}\left(\boldsymbol{\mathcal { R }}_{0}<\mathbf{1}\right) \text { and } \boldsymbol{H}_{\mathbf{2}} \\
\text { do not hold, otherwise it is a saddle point }\end{array}$ \\
\hline$E_{4}\left(S_{4}, I_{4}, 0,0\right)$ & If $\boldsymbol{H}_{1}$ holds. & $\begin{array}{c}\text { Saddle point, dimension of unstable manifold } \\
\text { is two if } \boldsymbol{H}_{\mathbf{4}} \text { holds and one otherwise. }\end{array}$ \\
\hline$E_{6}\left(S_{6}, I_{6}, K, 0\right)$ & If $\boldsymbol{H}_{\mathbf{1}}$ holds. & $\begin{array}{l}\text { Locally asymptotically stable if } \boldsymbol{H}_{\mathbf{5}} \text { does not hold, } \\
\text { otherwise saddle point with one-dimensional unstable manifold. }\end{array}$ \\
\hline$E_{7}\left(K, 0, N_{7}, P_{7}\right)$ & If $\boldsymbol{H}_{\mathbf{2}}$ holds. & $\begin{array}{l}\text { Locally asymptotically stable if } \boldsymbol{H}_{10} \text { holds }\left(\boldsymbol{\mathcal { R }}_{2}<\mathbf{1}\right) \text {. } \\
\text { otherwise saddle point with one-dimensional unstable manifold }\end{array}$ \\
\hline$E_{81}\left(S_{81}, I_{81}, 0, P_{81}\right)$ & $\begin{array}{l}\boldsymbol{H}_{\mathbf{4}} \text { does not hold } \\
\text { and } \boldsymbol{H}_{\mathbf{8}} \text { holds }\end{array}$ & $\begin{array}{c}\text { Stable if all roots of the characteristic equation, } \\
\text { of } \boldsymbol{J}_{\mathbf{8 1}} \text { have negative real parts. }\end{array}$ \\
\hline$E_{82}\left(S_{82}, I_{82}, 0, P_{82}\right)$ & $\begin{array}{c}\text { Either } \boldsymbol{H}_{\mathbf{4}} \text { holds or } \\
\boldsymbol{H}_{\mathbf{4}} \text { does not hold and } \boldsymbol{H}_{\mathbf{8}} \text { holds }\end{array}$ & $\begin{array}{l}\text { Stable if all roots of the characteristic equation, } \\
\text { of } \boldsymbol{J}_{\mathbf{8 1}} \text { have negative real parts. }\end{array}$ \\
\hline$E_{*}\left(S_{i *}, I_{i *}, N_{i *}, P_{i *}\right)$ & See $\boldsymbol{H}_{\mathbf{9}}$ & $\begin{array}{l}\text { Stable if all roots of the characteristic equation, } \\
\text { of } \boldsymbol{J}_{i *} \text { have negative real parts. }\end{array}$ \\
\hline
\end{tabular}

TABLE 3. Summary of existence and stability criterion for the equilibria of Model 2.

shows that its value decreases compared to the same model with a single prey (not shown here); this is due to an increase in the stationary value of $S$.

As in Model 1, a decrease in the carrying capacity $K$ in the current model results in the disappearance of domain $E_{8}$ in Fig.7: there is no extinction of prey $\# 2$ for some intermediate $\alpha$ and $\lambda_{0}$. We do not show the altered bifurcation diagram in this case for the sake of brevity.

Let us consider next the other possible situation, where the predator cannot survive by consuming prey \#1 alone. A typical bifurcation diagram is shown in Fig.8 (constructed for the same parameters as Fig.3). The overall structure of this diagram is close to that of the model with a specialist predator (cf. Fig.6, note that replacing $K=5$ does not change the portrait qualitatively). For example, Model 2 with $N \neq 0$ can exhibit bi-stability for small $\lambda_{0}$. Moreover, a large predator-dependent transmission $\alpha$ will result in a destabilization of the system: species densities begin oscillating around an unstable $E_{*}$ (this is denoted by L.C in the figure). The condition for disease establishment is the same as in the absence of prey $\# 2$, which is given by $\mathcal{R}_{0}>1$. On the other hand, the coexistence of all four components becomes enhanced in the presence of the second prey: the survival of the predator is observed for larger values of $\lambda_{0}$. Interestingly, although the population cycles in Fig.8 are not suppressed, adding a second prey results in a substantial drop in the amplitude of oscillations (by a factor of 8-10 for the given set of parameters), which is observed within a large range of $\alpha-\lambda_{0}$ (the corresponding graphs are not shown here). As a result, due to the existence of an alternative food source, the population density of prey \#1 does not exhibit a severe drop to very small values (as it would be for $N=0$ ), which would signify species extinction in a real ecosystem. Thus, the presence of prey \#2 enhances the persistence conditions of prey \#1 and the predator.

Finally, we should say that after completing a rather extensive investigation, we could not find a single set of parameters, corresponding to the triple exterior equilibrium $\left(S_{*}, I_{*}, N_{*}, P_{*}\right)$ with all positive 


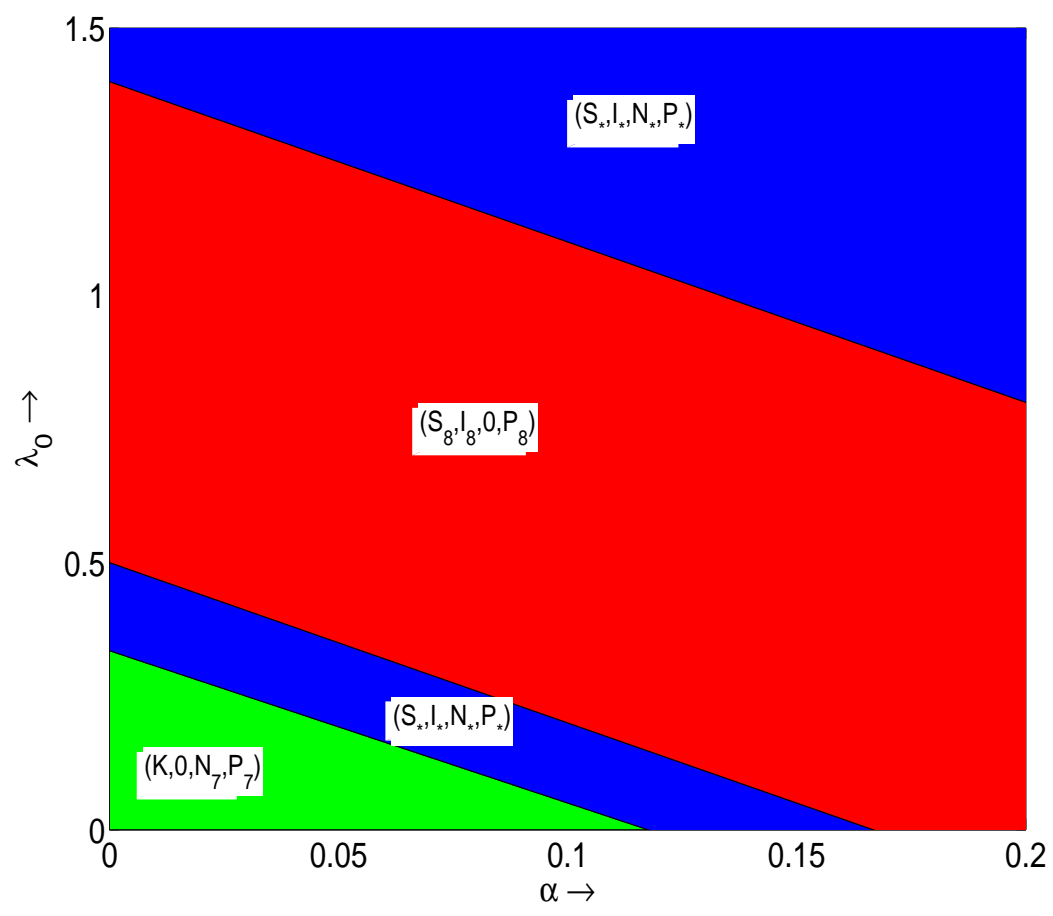

Figure 7. Bifurcation diagram of Model 2 in $\alpha-\lambda_{0}$-plane for the parameter set: $r_{1}=1$, $K=10, a=1, r_{2}=3, b=1, \theta_{1}=.2, \theta_{2}=.2, d_{2}=.1$.

densities. This might signify that even if such a parameter set were to exist, the domain size should be rather narrow. Finding such a parameter set can be an important future extension of the current work.

\section{Discussion and conclusions}

Most models in theoretical eco-epidemiology make the important assumption that the predator consumes only one prey species (see references in [35]). This is obviously a too simplistic scenario and the current paper is suggested to partially bridge the existing gap and explore the role of an alternative prey in the disease spread and overall ecosystem dynamics. A number of publications consider the scenario where the predator consumes both susceptible and infected prey, and so formally the predator does have two sources of food: healthy and infected prey (see examples in [35]). However, the two subpopulations ( $S$ and $I$ ) of the same prey cannot be regarded as two separate species. Indeed, alteration in the growth rate/carryng capacity of the healthy subpopulation $S$ automatically affects the growth rate of the density of the infected subpopulation $I$. Moreover, extinction of $S$ would automatically result in an imminent extinction of $I$. On the other hand, in the existing few eco-epidemiological models, where the predator does have an alternative food source, such a source was always suggested to be fixed, i.e. to be 'static' $[14,15,30]$. In this paper, we consider the density of the alternative prey $N$ to be a dynamical variable. Finally, we explore here eco-epidemiological dynamics for two different scenarios of transmission rate: predatorindependent and predator-dependent disease transmission. The last scenario is largely overlooked in the literature.

After fulfilling a comprehensive bifurcation analysis, we find that the introduction of a second prey in the system can strongly affect the spread of the disease in the first prey species. This can be described in terms of the basic reproduction number of the infection. We have introduced two modified basic 


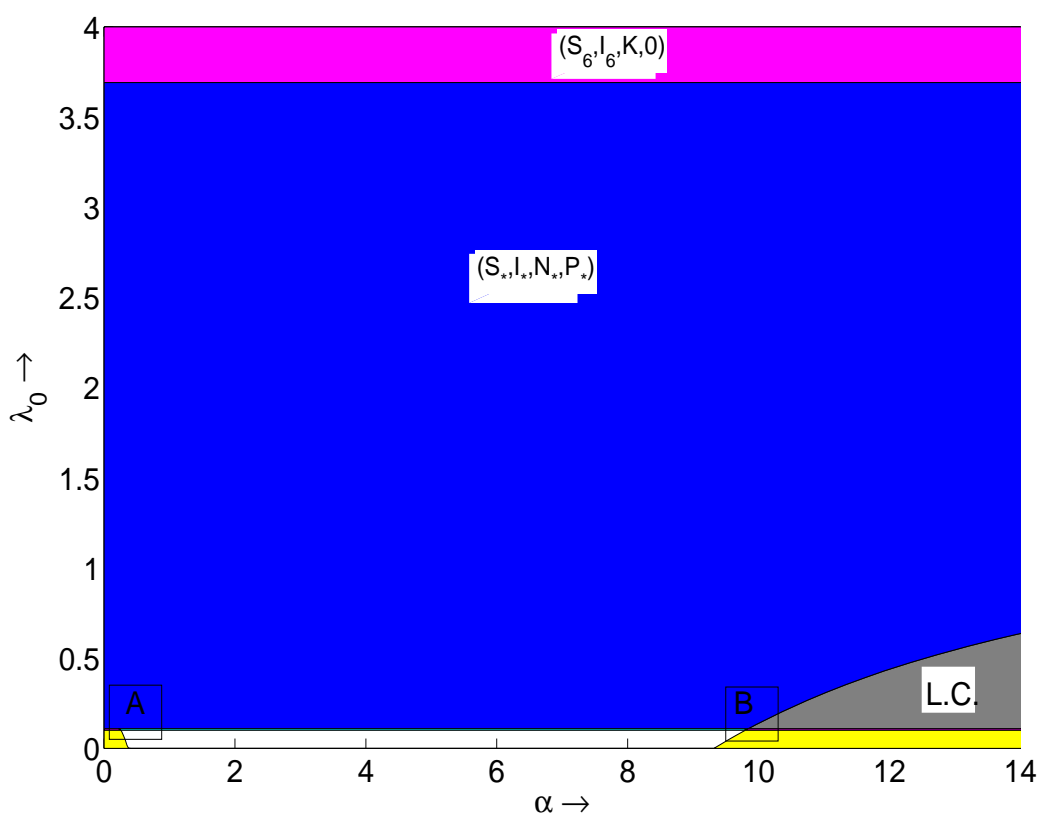

A

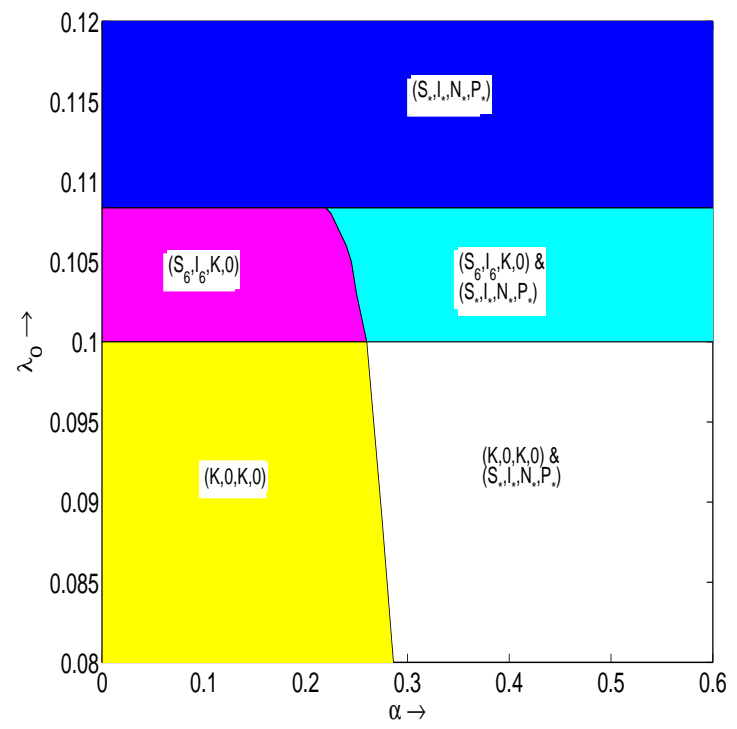

B

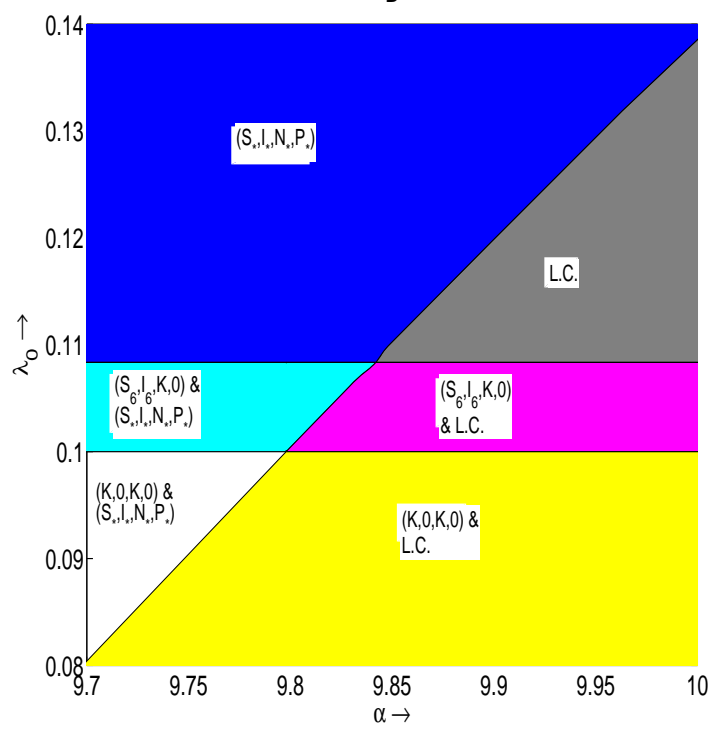

Figure 8. Bifurcation diagram of Model 2 in $\alpha-\lambda_{0}$-plane for the parameter set: $r_{1}=1$, $K=5, a=1, r_{2}=3, b=1, \theta_{1}=.2, \theta_{2}=.01, d_{2}=.1$.

reproduction numbers $\mathcal{R}_{1}$ and $\mathcal{R}_{2}$, which depend on the predator density (see (3.7) and (4.11)), these numbers generally provide more restrictive conditions for disease spread than $\mathcal{R}_{0}$ (i.e. establishment of the infection requires a larger transmission rate). However, they are valid only in the case where the alternative prey can support the growth of the predator population alone: otherwise the condition for 
infection spread is determined by $\mathcal{R}_{0}$. Furthermore, computation of the disease prevalence shows that including a second prey can substantially decrease its value (Fig.3). Mathematically, this follows from the equation for the infected prey $I$ : at equilibrium a higher density of predator $P$ - due to the presence of an alternative resource - should be compensated by a higher density of healthy subpopulation $S$, which should decrease the disease prevalence $I /(S+I)$, since the value of $I$ varies only slightly. Thus, the infection prevalence predicted using a single prey model can be somewhat overestimated, which is an important consideration for practical disease management in ecosystems.

We show that the presence of an alternative prey enhances the persistence of the predator regardless of whether or not prey \#2 can support the predator population alone. This is true for both transmission scenarios, and can be seen from a comparison of the corresponding bifurcation diagrams obtained for the specialist and the generalist predators. On the other hand, the spread of disease in prey \#1 can strongly affect the population density of prey $\# 2$, and can even cause it to go extinct (e.g. figs.2,7). For example, for high values of carrying capacity $K$, coexistence between the two prey species is impossible for an intermediate range of disease transmission rate. Since the prey species do not interact directly, this is an example of strong apparent competition [32].

Recently it was shown that predator-dependent disease transmission can result in system bi-stability and destabilization even for a linear predator functional response [25]. Our work shows that the previous findings are not justified in the case of a generalist predator, where the second prey constitutes an important food source (i.e. the predator can survive solely by consuming the alternative prey). For instance, there is no Allee -effect like behaviour or species oscillations (see Fig.7), as was the case for a single prey model [25]. Paradoxically, even if the second prey cannot establish in the system in the long term and therefore formally the equilibrium species densities $S, I, P$ are equivalent to the model with a single prey, the overall system behaves differently to the case of a specialist predator, where the second prey is always absent. This happens because the equilibrium with $P=0$ is stable only in the subspace with $N \equiv 0$ and adding any amount of $N>0$ breaks down the stability. Since in nature there often exists some alternative prey species (even at low densities) we will always have $N>0$ and the equilibrium with $P=0$ will be unstable in the considered scenario. We also find that the presence of a second prey can dampen high amplitude oscillations in Model 2 caused by the predator-dependent disease transmission.

Here we consider two different disease transmission scenarios and it is worth comparing the overall effect of introducing a predator-dependent transmission to the system with a generalist predator. A thorough comparison between the two scenarios should be done elsewhere since it would require the construction of all possible parametric portraits. Our current findings show that inclusion of $\lambda(P)$ generally facilitates the spread of disease (provided $\alpha>0$ ). Adding $\lambda(P)$ can promote bi-stability in the system and oscillations of species densities in the case the second prey cannot support the predator population alone (cf. Fig.8). Also, introducing predator-dependent transmission can shift persistence conditions for the second prey (see Fig.7, domain $E_{8}$ ), i.e. either to result in extinction of $N$, or facilitate its survival depending on the conditions for $N$ at $\lambda(0)$.

Finally, we should mention that our study leaves some open questions. For instance, it would be an important extension to investigate the behaviour of the same model for a more realistic functional response with saturation (e.g. Holling type II). Also, it would be interesting to explore the possibility of more complex dynamics in Model 2, in particular, to investigate the possibility of having three coexisting interior equilibria. Another important future extension would be the use of a parametrization of the transmission rate other than the mass action term $\lambda S I$, in particular the frequency-dependent pathogen transmission which is widely used in the modelling literature [22,24]. We shall address these issues in following papers.

Acknowledgements. We thank Professor S. Petrovskii (University of Leicester, UK) and Professor E. Venturino (Universita' di Torino, Italy) for helpful discussions and comments. The work of M. Sen is partially supported by Dr. D. S. Kothari posdoctoral fellowship, UGC, India. 


\section{References}

[1] S. Altizer, C.L. Nunn, P.H. Thrall, J.L. Gittleman, J. Antonovics, A.A. Cunningham, A.P. Dobson, V. Ezenwa, K.E. Jones, A.B. Pedersen, M. Poss, J.R.C. Pulliam. Social organization and parasite risk in mammals: integrating theory and empirical studies. Annu. Rev. Ecol. Evol. Syst., 34 (2003): 517-547.

[2] R.M. Anderson, R. M May. Population biology of infectious diseases part I. Nature 280 (1979) 361-367.

[3] R. M. Anderson, R. M. May. The invasion, persistence and spread of infectious diseases within animal and plant communities. Philos. Trans. R. Soc. London B, 314 (1986) 533-570.

[4] M. Begon, J.L. Harper, C.R. Townsend. Ecology. Oxford, Blackwell Science, 2002.

[5] I. Cote, R. Poulin. Parasitism and group size in social animals: a meta-analysis. Behavioral Ecology, 6 (1995) $159-163$.

[6] O. Diekmann, J.A.P. Heesterbeek, J.A.J. Metz. On the definition and the computation of the basic reproduction ratio R0 in models for infectious diseases in heterogeneous populations. J. Math. Biol., 28 ((1990)) 365-382.

[7] M.A. Duffy, L. Sivars-Becker. Rapid evolution and ecological host- parasite dynamics. Ecol. Lett., 10 (2007) $44-53$.

[8] A. El-Gohary, A.S. Al-Ruzaiza. Chaos and adaptive control in two prey, one predator system with nonlinear feedback. Chaos Solitons \& Fractals, 34 (2007) 443-453.

[9] M. Friend. Avian disease at the Salton Sea. Hydrobiologia, 473 (2002) 293-306.

[10] S. Gakkhar, R.K. Naji. Existence of chaos in two-prey, one-predator system. Chaos Solitons \& Fractals, 17 (2003) 639-649.

[11] W. Gentleman, A. Leising, B. Frost, S. Strom, J. Murray. Functional responses for zooplankton feeding on multiple resources: a review of assumptions and biological dynamics. Deep Sea Res. II, 50 (2003) 2847-2875.

[12] M.E. Gilpin. Spiral chaos in a predator-prey model. Am. Nat., 113 (1979) 306-308.

[13] K.P. Hadeler, H.I. Freedman. Predator-prey population with parasitic infection. J. Math. Biol., 27 (1989) $609-631$.

[14] M. Haque, E. Venturino. The role of transmissible diseases in the Holling-Tanner predator-prey model. Theor. Pop. Biol., 70 (2006) 273-288.

[15] M. Haque, E. Venturino. Effect of parasitic infection in the Leslie-Gower predator-prey model. J. Biol. Sys., 16 (2008) $445-461$.

[16] V. Hutson, G.T. Vickers. A criterion for permanent coexistence of species, with an application to a two-prey onepredator system. Math. Biosci., 63 (1983) 253-269.

[17] P.T.J. Johnson, D.E. Stanton, E.R. Preu, K.J. Forshay, S.R. Carpenter. Dining on disease: how interactions between infection and environment affect predation risk. Ecology, 87 (2006) 1973- 1980.

[18] Z. Kabata. Parasites and diseases of fish cultured in the tropics. London, Taylor and Francis, 1985.

[19] A. Klebanoff, A. Hastings. Chaos in One-Predator, Two-Prey Models: General Results from Bifurcation Theory. Math. Biosci., 122 (1994) 221-233.

[20] B. Krasnov, I.S. Khokhlova, G.I. Shenbrot. The effect of host density on ectoparasite distribution: an example of a rodent parasitized by fleas. Ecology, 83 (2002) 164-175.

[21] R. Kortet, M.J. Rantala, A. Hedrick. Boldness in anti-predator behaviour and immune defence in field crickets. Evol. Ecol. Res., 9 (2007) 185-197.

[22] H. Malchow, F.M. Hilker, S.V. Petrovskii, K. Brauer. Oscillations and waves in a virally infected plankton system. I. The lysogenic stage. Ecol. Compl., 1 (2004) 211-223.

[23] C. Matz, S. Kjelleberg. Off the hook - how bacteria survive protozoan grazing. Trends Microbiol, 13 (2005) $302-307$.

[24] H. McCallum, N. Barlow, J. Hone. How should pathogen transmission be modelled? Trends Ecol. Evol. 16 (2001) 295-300.

[25] A.Y. Morozov. Revealing the role of predator-dependent disease transmission in the epidemiology of a wildlife infection: a model study. Theor. Ecol., 5 (2012) 517-532.

[26] A. Morozov, A. Best. Predation on infected hosts promotes evolutionary branching of virulence and pathogen biodiversity. J. Theor. Biol., 307 (2012) 29-36.

[27] E. Odum, G.W. BARRETT. Fundamentals of Ecology. Belmont, Thomson Brooks/Cole, 2004.

[28] C. Packer, R.D. Holt, P.J. Hudson, K.D. Lafferty, A.P. Dobson. Keeping the herds healthy and alert: implications of predator control for infectious disease. Ecol. Lett., 6 (2003) 797-802.

[29] M.C. Rigby, J. Jokela. Predator avoidance and immune defence: costs and trade-offs in snails. Proc. R. Soc. Lond. B Biol. Sci., 267 (2000) 171-176.

[30] S. Roy, J. Chattopadhyay. Disease-selective predation may lead to prey extinction. M2AS, 28 (2005) $1257-1267$.

[31] S. Sharma, G. P. Samanta. Analysis of a two prey one predator system with disease in the first prey population. Int. J. Dyna. Cont., (In press) 2014.

[32] S. Van Nouhuys, S., I. Hanski. Apparent competition between parasitoids mediated by a shared hyperparasitoid. Ecol. Lett., 3(2) (2000) 82.

[33] R. R. Vance. Predation and Resource Partitioning in One Predator - Two Prey Model Communities. Am. Nat., 112 (1978) 797-813.

[34] E. Venturino. The influence of diseases on Lotka-Volterra systems. Rocky Mount. J. Math., 24 (1994) $381-402$.

[35] E. Venturino Ecoepidemiology: a more comprehensive view of population interactions MMNP, (In press) 2015.

[36] E. Venturino, S. Petrovskii. Spatiotemporal behavior of a prey-predator system with a group defense for prey Ecol. Compl., 14 (2013) 37-47 